UCRL- 89841

PREPRINT

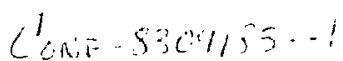

NUCLEOSYNTHESIS IN STELLAR EXPLOSIONS $\quad \cdots: \cdots 7: 7$

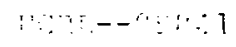

S. E. Woosley

Timothy S. Axelrod

Thomas A. Weaver

This paper was prepared for inclusion in

the Proceedings of the Erice Conference

on Nucleosynthes is

Erice, ITALY

September 9, 1983

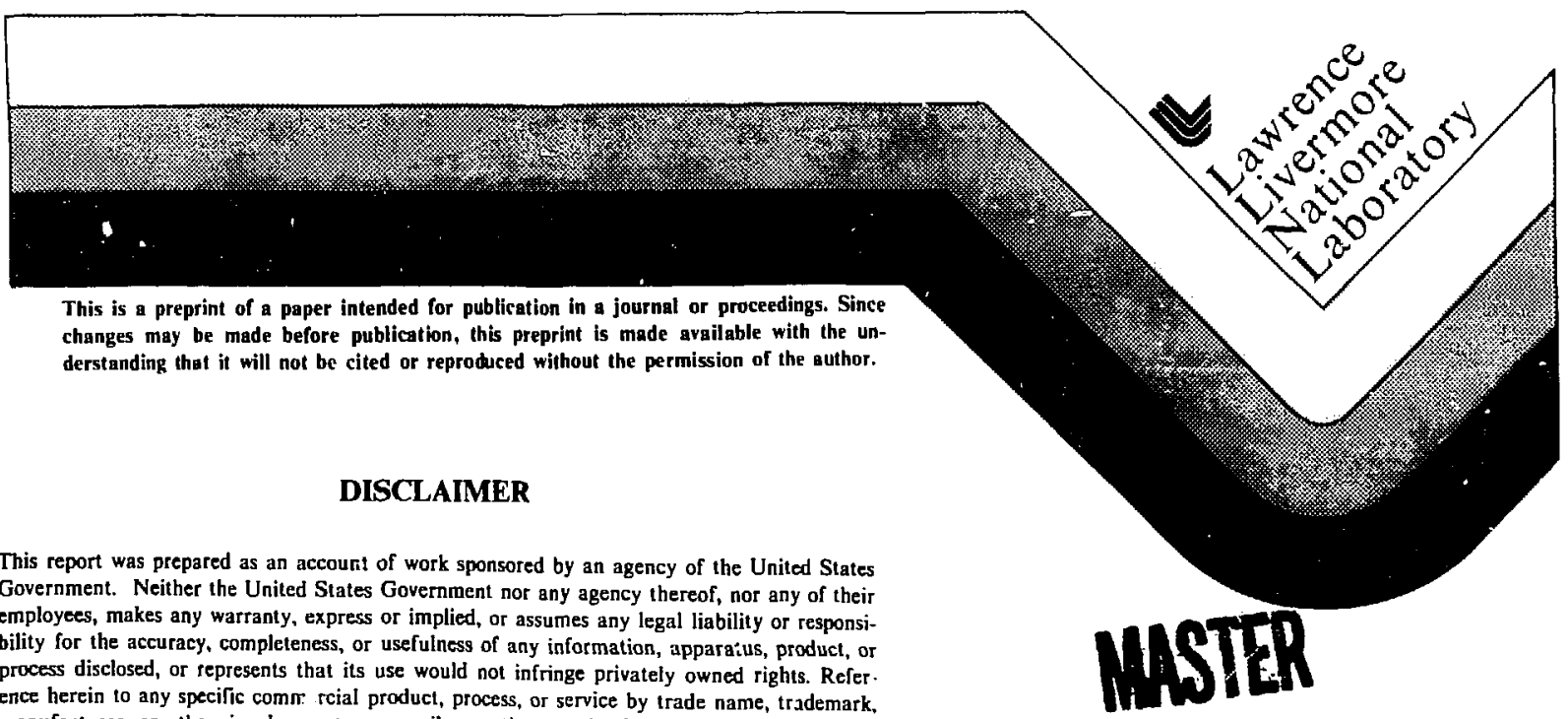
manufacturer, or otherwise does not necessarily constitute or imply its endorsement, recommendation, or favoring by the United States Government or any agency thereor. The views and opinions of authors expressed herein do not necessarily state or reflect those of the United States Government of any agency thercof.

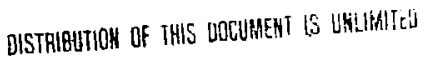




\title{
NUCLEOSYNTHESIS IN STELLAR EXPLOSIONS
}

\author{
S. E. Woosley \\ Board of Studies in Astronomy and Astrophysics \\ University of California, Santa Cruz 95064 , \\ and \\ Special Studies Group, Lawrence Livermore National Laboratory \\ University of California, Livermore CA 94550 , \\ Timothy S. Axelrod \\ T-Division, Lawrence I,ivermore National Laboratory \\ Iniversity of California, Livermore CA $\mathbf{9 4 5 5 0 ,}$ \\ and \\ Thomas A. Weaver \\ Special Studies Group, Lawrence Livermore National Laboratory \\ Eniversity of California, Livermore CA 94550
}

\begin{abstract}
The final crolution and explosion of stars from $10 \mathrm{M} \odot$ to $10^{\circ} \mathrm{M}_{\odot}$ are reviewed with emphasis on factors affecting the expected nuclcosynthesis. W'e order our paper in a sequence of decreasing mass. If, as many suspect, the stellar birth function was peaked towards larger masses at earlier times (see e.g., Silk 1077; but also see Palla, Salpeter, and Stahler 1983), this sequence of masses might also be regarded as a temporal sequence. At each stage of Galactic chemical evolution stars form from the ashes of preceding generations which typically had greater mass. $A$ wide variety of Type I supernova models, most based upon accreting white dwarf stars, are also explored using the expected light curves, spectra, and nucleosynthesis as diagnostics. No clearly favored Type I model emerges that is capable of simultaneously satisfying all three constraints.
\end{abstract}

\section{PATICE:}

POPTIONE UF THIS REPDRT ARE ILLEGBLE.

it has beon reproduced from the besi availante copy to pormit the broatest possitsie availability.

OISTRIBUTOH OF THLS OOCUMENT IS UHLIMUTED

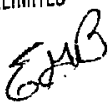




\section{SUPERMASSIVE STARS $\left(\mathrm{M}>10^{5} \mathrm{M}_{\odot}\right)$}

The most massive stars to form early in the Universe might have had masses on the order if the Jeans' mass at the time of radiation decoupling, $\sim 10^{6}$ to $10^{\circ} \mathrm{M}_{\odot}$, coincidentally the mass of : typical globular cluster (Dicke and Peebles 1968). Aiternatively such supermassive objects micht. have formed at a later time, and with a greater metai city, from the relaxation of a dense : t: llar cluster (Sanders 1970). Stars having such great mass are general relativistically unstabie (iben 1963; Fowler 1966). After spending only a few thousand years contracting as gravity provides the radiation leaving their surface (Kelvin-Helmholtz stage), the stars reach a critical radius. $\lesssim$ $10^{14} \mathrm{~cm}$ for a star of $5 \times 10^{6} \mathrm{M}_{\odot}$, where the first order relativistic corrections to Nowtonian gravity render the star, which has $\gamma$ very nearly equal to $4 / 3$, unstable to continuing collapse. Were it not for the presence of unburned nuclear fuel this collapse would continue indefinitely until the star became a black hole. Because the star does contain hydrogen, however, it is possible for nuclear reactions during the implosion to release sufficient energy to power a gigantic explosion.
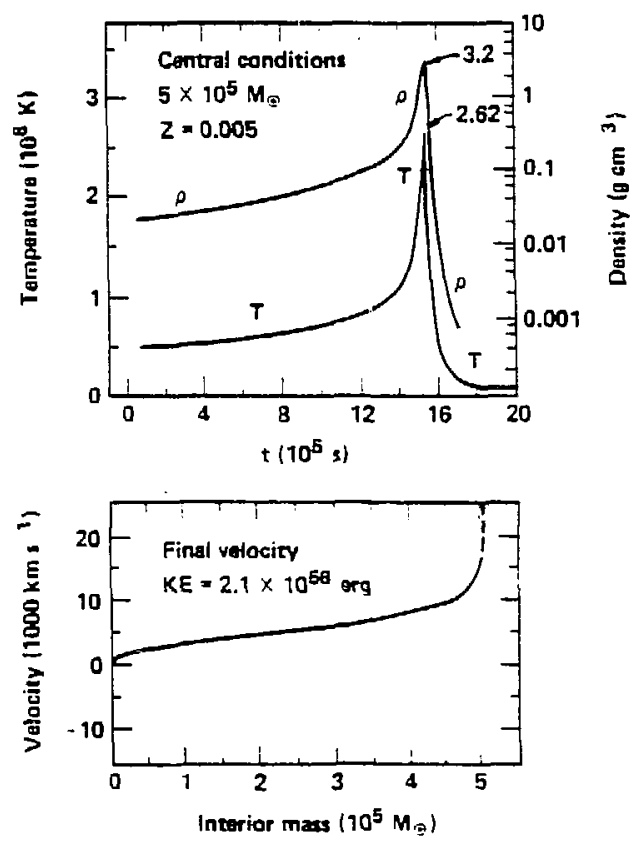

Figure 1. Thermodynamic conditions and final expansion velocity in a supermassive stellar explosion. Peak values of central temperature (in units of $10^{8} \mathrm{~K}$ ) and density $\left(\mathrm{g} \mathrm{cm}^{-3}\right)$ are indicated. The velocity curve is dashed in the outermost layers where the mass zoning was not adequate for an exact calculation. The kinetic energy is given at infinity. 
Fuller, Woosley, and Weaver $(1982,1883)$ have recently constructed numerical models of supermassive stars in the range $10^{5}$ to $10^{\circ} \mathrm{M} \odot$ using a one-dimensional implicit hydrodynamics code (KEPLER; Weaver, Zimmerman, and Woosley 1978) revised to include first order post-Newtonian corrections to gravity and nuclear burning by the rp-process (Wallace and Woosley 1981). They find, similar to Fricke (1973, 1974); Appenzeller and Fricke (1972); and Appenzeller and Tscharnuter (1973). but contrary to Ober (1981), that non-rotating stars in this mass range will collapse to black holes with no reversal of implosion unless the initial metallicity of the star is already a substantial fraction of solar (i.e., $Z \gtrsim 0.005$ ). The rsults are quite sensitive to the metallicity since the rate of hydrogen burning is proportional to the abundances of catalytic nuclei in the $\mathrm{C} \mathrm{NO}$ group. Lower burning rates do not release sufficient energy during the infall to reverse the implosion. Supermassive stars having mass $1 \times 10^{5}(Z=0), 2.5 \times 10^{6} \quad(Z=0), 5 \times 10^{5} \quad(Z=0$, $0.002,0.005,0.01)$, and $10 \times 10^{5}(Z=0,0.006,0.01)$ solar masses were studied. The $10^{5} \mathrm{M}_{\odot}$ star burned hydrogen in a stable fashion, i.e., it did not experience the general relativistic instability prior to hydrogen depletion in the center of the star and it did not collapse. All other models with the exception of three produced black holes. The three exceptions were two $5 \times 10^{5} \quad M_{\circ}$ stars with metallicity 0.005 and 0.01 , and one $10^{\circ} \mathrm{M}$ star with metallicity 0.01 . These models. completely disrupted with kinetic energies of $\cdots 10^{50}$ to $10^{57}$ erg. The central temperature and density history during the implosion/explosion of one $5 \times 10^{5} \mathrm{M}_{\odot}$ model is shown in Figure 1 . In the $10^{\circ} \mathrm{M}$ explosion the luminosity, which during the Kelvin-Helmholtz stage was near $10^{44}$ erg s $\mathrm{s}^{-1}$, i.e., the Eddington value, rises to $\gtrsim 10^{45} \mathrm{erg} \mathrm{s}^{-1}$ and stays that bright for approximately 10 years. Apparently if such gigantic stars ever do form they will make their presence known!

The chief nucleosynthetic consequences of these explosions are the production of helium from hydroger and trace amounts of ${ }^{15} \mathrm{~N}$ and ${ }^{7} \mathrm{Li}$ (Wallace and Woosley 1981; Norgaard and Fricke 1976). The helium nass fraction in both $5 \times 10^{5} \mathrm{M}$ explosions rises by $3^{\circ} \mathrm{c}$ and in the $10^{\circ} \mathrm{M}_{\circ}$ explosion, it rises by $16 \%$. One should keep in mind, however, that were it not for a near solar concentration of heary elements these stars would never have exploded in the first place. Thus non-rotating supermassive stars (heavier than $10^{5} \mathrm{M}=1$ played absolutely no role in producing pre-cialactic helium.

Quite different results may be expect $f$ if these stars are endowed with rotation (Fowler 1966; Fricke 1974). In particular, higher central temperatures may be reached by objects that have lower initial metallicity (zero ?) yet succeed in exploding because of the centrifugal potential. At these higher temperatures the rp-process can produce heavy elements up to the iron group and beyond (Wallace and Woosley 1981). Fuller et al intend to nodel rotating supermassive stars during the forthcoming year by introducing a one-dimensional "pseudo-rotational" potential into the same KEPLER code used to study the non-rotating models. Full two-dimensional calculations should eventually be attempted. 


\section{VERY MASSIVE STARS ("HYPERNOVAE")}

Stars forming with initial masses in the 'ange roughly 100 to $300 \mathrm{M}_{\bullet}$ (without rotation) $r \mathrm{x}$ perience an instability that bears certain similarities to the supermassive stars discussed abowe Following stages of relatively stable hydrogen and helium burcing (during which pulsational ibstabilities exist that may drive rapid mass loss, Stothers and Simon 1970; Talbot 1971; Papaloizi:: 1973) the stellar core enters a thermodynamic regime that favors the creation of large quari' $i$ ties of electron-positron pairs. Creation of these pairs takes energy that might otherwise have provided pressure support and thus the star collapses (Barkat, Rakavy, and Sack 1967). again with $\gamma$ close to $4 / 3$. Nuclear fusion during the collapse liberates energy from carbon, neon, and oxygen burning, but silicon burning requires energy. This is because, at the low densities characteristic of such massive stars, silicon burning produces ${ }^{54} \mathrm{Fe}+2 \mathrm{p}$, not ${ }^{50} \mathrm{Ni}$. and the net reaction sequence is endoergic. If sufficient energy is liberated during the collapse by carhon, neon and oxygen burning then the star blows up; but if the implosion continues well into siliern burning (and retation is negligible), a h.ck hole forms. Consequently. non-rotating stars having mass greater than about $300: 1=$ (and less than $10^{5} \quad \mathrm{M}$ ) end up as black hole: Below $100 \mathrm{M}$. the pair instability is not encountered and the star undergoes stable carbon, neon, oxygen. and silicon burning, culninating in iron core collapse (see next Section).

In the range 100 to $300 \mathrm{M}$ (these being rougn estimates of the mass of the star at its birth on the main sequence prior to mass lossi, stars end their lives in gigantic explosions called "hypernovae". Such stars have been recently modeled by Woosley and Weaver (1982a); Bond, Arnett, and Carr (1982. 1983; see also Bond. this volume); and Ober, El Eid, and Fricke (1983). The evolution of a $200 \mathrm{M}_{\odot}$, Pop III (i.e. $Z=0$ ) star studied by Woosley and Weaver is typical. This star burns hydrogen at a central temperature of $\sim 1.2 \times 10^{8} \mathrm{~K}$ and density $\sim 20 \mathrm{~g} \mathrm{~cm}^{-3}$ for $\sim 2.5$ million years (note that the main sequence lifetimes of stars in this mass sange are approximately constant owing to the Eddington limitation on their luminusities and the fact that they are convective throughout most of their mass). The luminosity of the $200 \mathrm{M}$ star on the

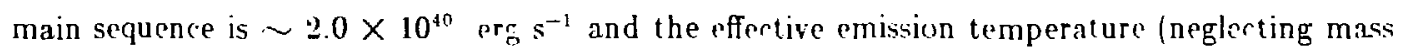
loss) is $80,000 \mathrm{~K}$. Because the luminosity is so close to Eddington and the core pulsationally unstable, extensive mass loss may well modify the surface properties leading to softer, perhaps even infra-red emission. It is interesting that hydrogen burning in this star is mediated by the CNO-cycle despite the fact that the star was born with no metals. Prior to hyc'rogen ignition the star contracts, heats up and actualiy burns a trace of helium to nitrogen ( $210^{-0}$ by mass) before igniting hydrogen burning (see also Ezer and Cameron 1971). At one-half bydrogen depletion the nitrogen mass fraction is $\sim 10^{-8}$.

Following hydrogen exhaustion, the $200 \mathrm{M}_{\odot}$ star burns belium for 265,000 years at a central temperature near $2.5 \times 10^{8} \mathrm{~K}$ and density $250 \mathrm{~g} \mathrm{~cm}^{-3}$. During this time the radius of the star 
expands greatly to $\sim 10^{14} \mathrm{~cm}$. At the end of helium burning, the inner $112 \mathrm{M}_{\odot}$ is comprised of oxygen and carbon in a roughly 3 to 1 ratio with traces of neon and magnesium. As the star contracts and heats in its center to $\sim 10^{9} \mathrm{~K}$, seeking to ignite the ${ }^{12} \mathrm{C}+{ }^{12} \mathrm{C}$ reaction, it encounters the pair instability and begins to collapse rapidly (although not super-sonically and $\mathrm{nut}$ in free fall). The central temperature rises to $3.9 \times 10^{\circ} \mathrm{K}$ and density to $2.2 \times 10^{\circ} \mathrm{g} \mathrm{cm}^{-\mathrm{s}}$ as carbon burns in the inner $\sim 80 \mathrm{M}_{\odot}$, neon in the inner $\sim 70 \mathrm{M}_{\odot}$, and oxygen in the inner $\sim$ $20 \mathrm{M} \approx$. The energy released by this burning is ade?uate to explode the star with total kinetic energy $2.6 \times 10^{52}$ erg. For 140 days the light output of this hypernova is $\sim 10^{44} \mathrm{erg} \mathrm{s}^{-1}$, or about 10 times that of a typical Type II supernova (see Woosley and Weaver 1982a for light curve).

The nucleosynthesis from this explosion is summarized in Table 1 with results normalized to solar system abundances (Cameruū 1973 ) relative to ${ }^{10} \mathrm{O}$, an abundant product of the explosion. The lack of iron production is a commun trait of this class of models owing to the endoergic nature of silicon burning. It is interesting that these explosions are such efficient producers of heavy elements (more than $1 / 2$ of the initial mass ends up in elements heavier than helium) that the explosion of only one or two could account for the abundances of elements from carbon through calcium found in a typical globular cluster of stars $\left(10^{\circ} \mathrm{M}, \mathrm{Z} \sim 10^{-4}\right)$. Ten thousand such explosions could produce a metallicity throughout the Galaxy comparable to that observed in the most metal-deficient st.ars (eg. CD-38-245, Bessel and Norris 1981; see also Bond 1981). If this did in fact occur ai very early times, one might expect to find, in the most metal deficient stars, large overabundances of the elements from carbon through calcium relative to iron (compared to the sun). Observations indicate that such a correlation may indeed exist (Sneden. Lambert, and Whitaker 1979; Butler, Dickens and Epps 1978; Bessel and Norris 1981). Unless some unforeseen circumstance prohibited star formation in this mass range or led to dispersal of the star prior to the pair instability, it is hard to see how the nucleosynthetic contribution from stars in this mass range could have been anything other than very important.

Table 1: Nucleosynthesis in a $200 \mathrm{M}_{\odot}$ Pop III Star

\begin{tabular}{|c|c|c|c|c|c|}
\hline Species & ${ }^{4} \mathrm{He}$ & ${ }^{12} \mathrm{C}$ & ${ }^{10} \mathrm{O}$ & ${ }^{20} \mathrm{Ne}$ & ${ }^{24} \mathrm{Mg}$ \\
\hline Mass ejected $\left(\mathrm{M}_{\mathrm{O}}\right)$ & 70 & 11 & 57 & 4.5 & 5.5 \\
\hline Compared to sun & 0.03 & 0.45 & 1 & 0.44 & 1.7 \\
\hline Species & ${ }^{28} \mathrm{Si}$ & ${ }^{32} \mathrm{~S}$ & s"Ar & ${ }^{40} \mathrm{Ca}$ & ${ }^{66} \mathrm{Fe}$ \\
\hline Mass ejected $\left(M_{\diamond}\right)$ & 20 & 6.8 & 0.85 & 0.66 & 0.08 \\
\hline Compared to sun & 4.6 & 2.7 & 1.4 & 1.4 & 0.01 \\
\hline
\end{tabular}


Other important implications for stars in the mass range $>100 \mathrm{M} \odot$, especially for providing "missing mass", helium synthesis, and microwave background are discussed by Bond "linuhur" in this volume (see also Bond. Arnett, and Carr 1982, 1883). We would only briefly inment on work in progress by Stringfellow. Woosley, and Bodenheimer regarding the evolution ef very nassive stars including rotation. As noted above the fate of non-rotating stars having ri: in siquence mass greater than $-300 \mathrm{M} \approx$ is a black hole. Stringfellow recently examined a $300 \mathrm{M}$ helium core (corresponding to a main sequence mass of $\geq 500 \mathrm{M}$ ) using a twodimmsinnal explicit hydrodynamics code (Bodenheimer anu Woosley 1983). The 300 M core was first evolved

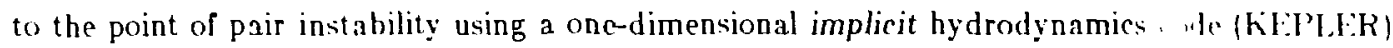
then remap $d$ onto the two-dimensional grid at a time when its central temperature had reached $2 \times 10^{\circ} \mathrm{K}$. Continued evolution in one dimension led to the formation of a black hole. This same evolution to black hole was replicated in the 2-D code if the star "as not rotating. Stringfrllow found, however, that the implosive crolution is greatly modified by the introduction of only a small amount of rotation. A rotational energy corresponding to 0.47 of the gravitatumal buntums energy at the beginning of the calculation (i.e.. when the central temperature wa $2 \times 10^{\circ} \mathrm{K}$ and

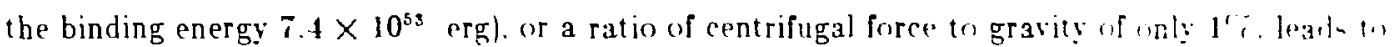
a reversal of the implosion and a gigantic explosion. During this explosi n mater at the center uf the star reaches 12 billion deg*ees and the central density rises to $4 \times 10^{-} \mathrm{g} \mathrm{cm}^{-}$. num hreater than in any of the one-dimensional calculations. A similar calcularion employ ing 5 times as much rotational energy also explodes but reaches a somewhat lower temperature $\left(5.9 \times 10^{\circ} \mathrm{h}\right)$. ( nfortunately the nuclear physies included in these two-dimensional calculations (only oxygen burning) is not adequate for surh extreme thermodynamic enditions. In particular. photodisintegration. a very important endoergic process that can diminish the prospects for an explosion. was left out. Stringlellow is currently rewriting his program to include photodisinergration and electron capture and we should have reliable results in the ncar future. An interesting posibility is mass "bifurcation". i.e., the central portions of the star may continue to collapse while outre layers are ejected by a combination of nuclear burning and rotation (see also Bodenheimer and Wumaley 1983). Important implications loom for both nucleosynthesis and the "missing mass" problem. The higher explosion temperatures accessible with the inclusion of rotation may allow iron group nuclei to be synthesized and ejected. Ejection of only a small fraction of the stars mass as heary elements could eliminate the residual black bole as an important component of the missing mass.

\section{MASSIVE STARS (10 to $100 \mathrm{M}_{\odot}$ )}

Stars in the range 10 to $\sim 100 \mathrm{M} \odot$ end their lives by producing iron cores of roughly a Chandrasekhar mass which collapse owing to a combination of photodisintegration reactions (induced by the high core temperature) and electron capture (resulting from both the high core 
temperature and density). In the limited, although numerically significant mass range 8 to 10 11: the late evolution may be diverse and complex. This results, in part, from an inverted temptrature gradient set up by efficient neutrino cooling in a star whose central pressure is only marginally sinsitive to tamperature and, in part. from the semi-degenerate nature of the nuclear burning itself (Wiaver and Woosley 1979; Woosley, Weaver, and Taam 1980; Miyaji et al 1980; Vomoto, 1980a, 1981, 1983, this volume; Hillebrandt 1982a). Here we will restrict our attention to the (comparatively) simpler evolution of more massive stars.

Much has been written about the evolution of stars in this mass range and, especially, the core bunnce/neutrinu transport mechanism which may for may not, depending seemingly upon the year in which one lives!) lead to their explosion. We shall not, in this limited space, even attempt 11) revien the extensive literature dealing with this subject. The iaterested reader may consult revew by Arnett (1980): Trimble (1982); Wheeler (1981); Hillebrandt (1982b, 1983): Bowers and Wilon (1982ab); and Brown. Bethe. and Baym (1982). Here we will briefly discuss some recent remult - from nur ungoing attempt to numerically simulate the complete evolution of a grid of - tellar masses 10, 15. 20. 35. 50. and $100 . \mathrm{M}$. fof constant masil from birth on the main sequence through their death as Type II supernovae.

In 1978 Wiaver. Zimmerman. and Woosley published presupernova models of 15 and 25 . 11 thars. These were subsequently followed through simulated core bounce and explosion (Weaver and Woosley 1980:i) and yielded luminosity, radius, and effective iemperature histories in good agreenent with observations of typical Type II supernovae. Furthermore, the nucleosynthesis (alculated by Weaver and Woosley (1980a) and, subsequently in greater detail by Woosley and Weaver $(1082 \mathrm{~h})$, for the $25 \mathrm{M1}$ : star agreed reasonably well with solar system proportions of isotupes from " ${ }^{\prime}$ in on $\mathrm{Vi}$. There were notable deficiencies, however, as noted by the authors The atundanem of species in the silicon to titanium range were too small by roughly a factor "I i rimpured to nther impertant intopes such as ${ }^{10} \mathrm{O}$; " Va was overabundant by roughiy the ane fartor: and several intresting species: ${ }^{13} \mathrm{C}^{14.16} \times 1{ }^{10} \mathrm{~F} .{ }^{48} \mathrm{Ca}$, and ${ }^{47} \mathrm{Ti}$, were hardly produced at all hime uf these deficint productionn were not particularly worrisome. eg. ${ }^{13}\left(\mathrm{C}\right.$ and ${ }^{14}$

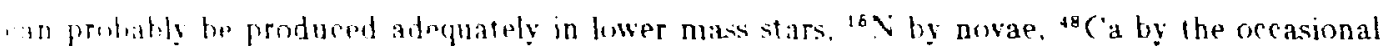
"Jutinn of highly neutronized core material, ete. but others. eg. the d.ficient production of sificon libnugh titanium, all of which are explosively synthesized during shock wave passage, led us to suggest that solar abundance set requires a component of nucleosynthesis from stars more massive than $25 \mathrm{M}_{\ominus}$. This is a proposition to which we still adhere for several reasons. First, as discussed in the previous section, stars of mass $\gtrsim 100 \mathrm{M}$ are copious producers of silicon through calcium (Table 1). C'nless nature has purposefully avoided their creation. hypernovae should form an important component of the nuclensynthetic matrix. Second, as one goes to higher mass stars the density gradient around the core becomes less steep. A shallower density gradient implies that 
the shock wave generated by core bounce (in stars less massive than $100 \mathrm{M}$ but more massive than $25 \mathrm{M}$ ) will heat more matter to peak temperatures in the range 3 to 4 billion degresi. i.e. the requisite range for explosive oxygen burning, and produce proportionately greatre amounts of silicon through calcium. Finally, it is unrealistic to expect the nuleosynthesis of all tare in the 10 to $100 \mathrm{M}$ s range to be so similar that element production in any one mass (eg. $25 \mathrm{M}$ / ir anything other than an extremely qualitative representation of the entire ensemble. An accurate calculation of iron group synthesis, for example, is an especially difficult problem as it relies (n) knowing both the strength of the shock and the location of the "mass cut" (last stellar zone to fall bark onto the neutron star) in stars of different total mass. Therefore. quantitative predurtions of iron synthesis are likely to remain highly uncertain for some time to come.

During the last several years we have computed the evolution of a number of of her mascive stars up to the point of silicon ignition. These include. in addition to the 1 ; 11 and $2:$ M

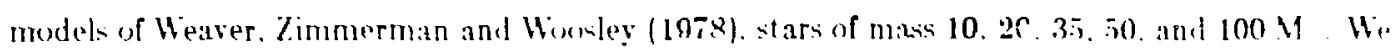
stopped these calculations at silienn ignition because the nuclear physics in the ende at that time.

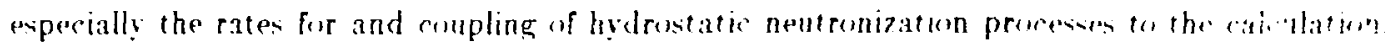

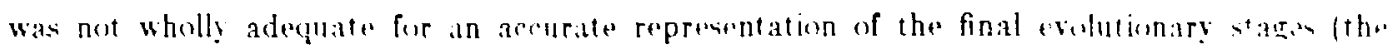

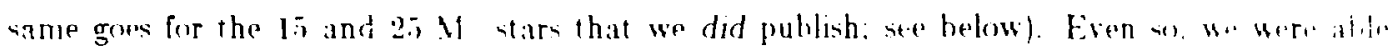
to de'ermine several interesting quantities that are not expected to be greatly modified during silicon core burning or by the ejection process. These include the helium rore mass at the end of the stars life: the mass of heavy $(Z>2)$ elements in the core (from which we can subtract roughly $1.5 \mathrm{M}$ : to obtain a rough estimate of the total mass of heavy elements ejerted by the star): and the nucleosynthesis of light isotopes $(Z \leq 12)$. Given the helium abundanee dredged up hy convection into the envelope. one can also estimate $J Y / L Z$. the change in helitim mass fraction divided by the chal o in metallicity. for these stars. The nucleusynthesis of light isotupess was reported in Woosle; and Weaver $(1982 a)$. The remaining quantities are summarized in Table 2. The metallieity 1.e. that fraction of the initial stellar mass finally ejerted in the form of elements heavier than helium. ran be approximatert in the nus. range 1.5 to 10011 by $7=0.5$ $-6.3 \mathrm{M}=/ \mathrm{M}$. 
Table 2: Bulk Nu-leosynthesis in Massive Stars

$\begin{array}{rrrrr}\text { Mins } & \begin{array}{r}\text { Helium Core } \\ (11 .)\end{array} & \begin{array}{r}\text { Metal Core } \\ (\mathrm{M})\end{array} & \mathrm{Y}_{\text {env }}{ }^{*} & \Delta Y / \Delta Z \\ 10 & 2.6 & 1.5 & 0.30 & \text { large } \\ 15 & 1.5 & 3.0 & 0.33 & 0.70 \\ 20 & 7.4 & 5.0 & 0.36 & 0.40 \\ 2.5 & 9.5 & 7.1 & 0.40 & 0.30 \\ 3.5 & 17.0 & 13.0 & 0.41 & 0.08 \\ 50 & 24.0 & 21.0 & 0.51 & 0.14 \\ 100 & 47.0 & 47.0 & 0.57 & 0.05\end{array}$

Initially the helium mass frartion in envelope was 0.28

More rerenty. Wiaver. Winsley and Fuller (1982. 1083: WWF) have revised the stellar wolufirn code so that the eleretron capture processes that commence during oxygen and silicon burning are properly modeled Mfudifications include weak interaction rates from Fuller, Fowler, and Vev:man (1982ab); a new treatment of quasiequilibrium and "cluster equilibrium" (Woosley, Arnett. and (layton 1973) that calculates the rate of neutronization (principally due to electron capture) during core and shell oxygen burning as well as during silicon burning and core collapse; new sedpences of nuclear reactions for silicon burning in a composition that has already become so "lintron-rich that ${ }^{3 \prime} \rightarrow$ is a major constituent: minor corrections to nuclear sereening fartors: initial ahundances from (ameron (1973) rather than Cox and Stewart (1970ab): and finer time step and Anilg riturta (roughly a factor of 2 in each). Esing the improved code, WWF have calculated 11.4 presupernuva models of 20 and $2.5 \mathrm{M}$ - stars. Other masses. eg. 10, 11, 15, 57, and $100 \mathrm{M}$. are in progress.

The rhis differencen in the new presupernora models (Figures 2 and 3) are smaller iron weres at the time of collapse: smaller values of $Y_{r}$. the electron mole number; and lower values of entropy lise WWF for plots of $Y_{e}$, and entropy in the new models). In 20 and $25 \mathrm{M}:$ pre supernova models the iron core masses are now 1.41 and $1.35 \mathrm{M}_{\odot}$ (as opposed to a value of 1.61 $\$ 1$ in the old $25 \mathrm{M}_{\odot}$ star); central values of $Y_{e}$ are 0.422 and 0.423 ; and central values of entropy are 0.60 and 0.73 respectively. The smaller cores and lower entropies should favor the prospects for explosion by the core bounce mechanism (eg. Hillebrandt 1982b; 1083). Additionally, the density gradients surrounding the iron cores of the new models are less steep. This implies, all else being equal. improved nucleosynthesis of elements from silicon through calcium. We plan to follow the (continued) explosive evolution of these new models in the near future. 


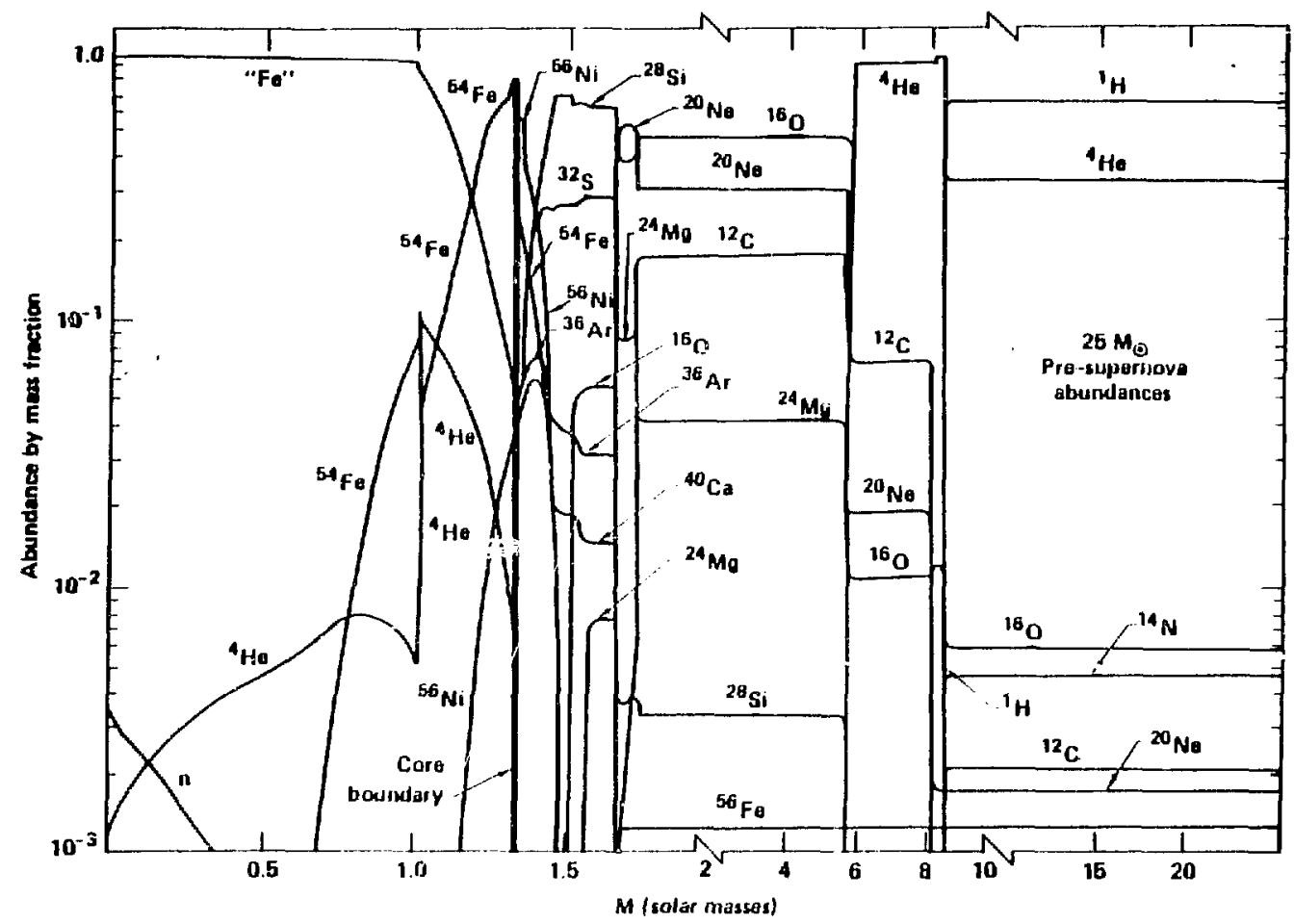

Figure 2. Abundances in the new $25 \mathrm{M}_{\odot}$ presupernova star (WWF). The composition is sampled at a time when the collapse velocity has lirst reached a value of $1000 \mathrm{~km} \mathrm{~s}^{-1}$ in any zone. Note the smaller iron core mass as compared to earlier calculations. Iforizonal abundance ines are in genera!, indicative of regions that are, or once were, convective. An exception is the curve labeled "Fe" which is the sum of the mass fractions of all iron group species more neutron-rich than stre. 


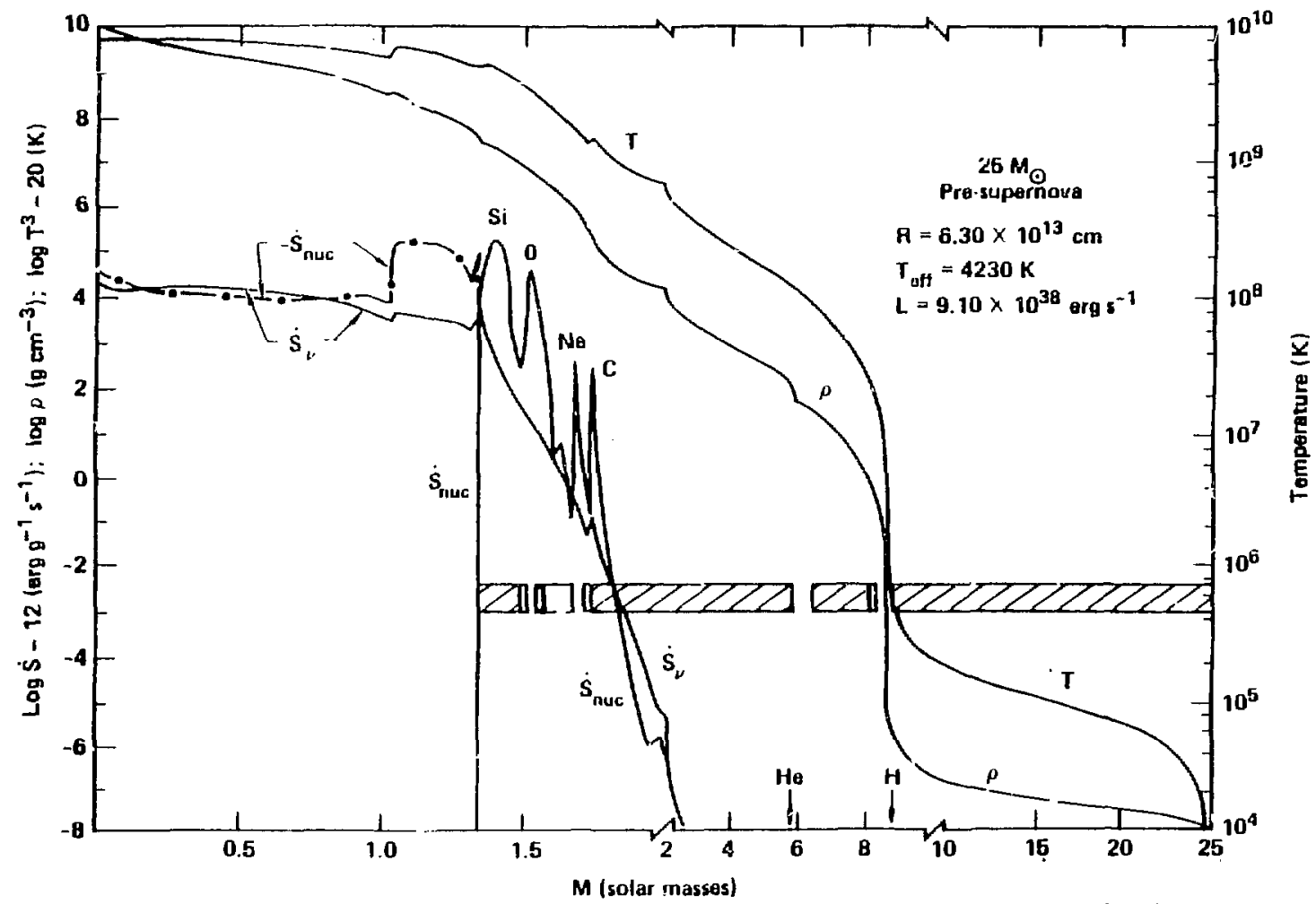

Figure 3. Thermodynamic conditions in the new $25 \mathrm{M}_{\odot}$ presupernova star (WWF). In the region external to the iron core the unclear energy gentration rate is positive and the neutrino loss rate negative. Internal to the core both are negative, with neutrino losses primarily coming from electron capture. Collapse here (at the same time as lighre 2) is a consequence of both photodisintegration and electron capture. Cross-hatched and open rectangles denote regions of convective and semi-convective instability respectively. Ilydrogen and heitum shells are burning at rates that are off-scale as indicated by arrows. Curves for $\rho$ and $T$ are parallel in reginns where the density scales $2 s T^{s}$. The stellar radius, effective emission temperature, and photon lamimsity are also given. 
Perhaps the most exciting recent development in studies of massive star evolution are the calculations of Wilson (1983) showing the delayed explosion of the collapsed core following a long period of shock stagnation. All the models Wilson has calculated now explode when the calculation is continued to sufficiently late ijmes. These include the "old" $10 \mathrm{M}_{\odot}$ star (see also Hillebrandt 198:a); the new $25 \mathrm{M}_{\odot}$ star (WWF); and the old $15 \mathrm{M}_{\odot}$ star (Weaver, Zimmerman, and W.cslcy 1978). Explosions occur at time ranging from $0.25 \mathrm{~s}\left(10 \mathrm{M}_{\odot}\right)$ to $0.55 \mathrm{~s}\left(25 \mathrm{M}_{\odot}\right)$; produce total explosion energies of from 1.8 to $4.5 \times 10^{60} \mathrm{erg}$; and leave neutron star masses (not corrected for neutrino losses) of $1.48 \mathrm{M}_{\odot}\left(10 \mathrm{M}_{\odot}\right), 1.76 \mathrm{M}_{\odot}\left(15 \mathrm{M}_{\odot}\right)$, and $1.66 \mathrm{M}_{\odot}\left(25 \mathrm{M}_{\odot}\right)$. Note that the $25 \mathrm{M}$ core accretes roughly $0.3 \mathrm{M}$ before finally exploding. The mechanism for these explosions involves the slow diffusion of neutrinos out of the core which capture on free nucleons behind an almost stationary accretion shock. The heating from this capture plus the diminished ram pressure as regions of decreasing density pass through the accretion shock (and the scattering of neutrin $s$ on pairs produced as the temperature rises and density drops, a lesser effect) ultimately combine to change the standing accretion shock into an outwardly propagating wave capable of ejecting the stellar mantle and envelope. Considerable numerical uncertainty exists in tiese calculations owing to the necessity of running thousands of time steps using explicit hydrodynamics and continuous mass rezoning. It will be very interesting to see the results of future calculations, perhaps employing implicit hydrodynamics and mixed Eulerian/LaGrangian mass zoning. Also interesting are the implications this all has for "leptonic convection" (Epstein 1979) at these late times and the possibility of core overturn (Colgate and Petschek 1979; Smarr et al 1981).

\section{TYPE I SUPERNOVAE}

There is gencral consensus that Type I supernovae (SNI) are the explosions of relatively low mass stars, at least compared to Type Il's, and that the stars involved are vid of substantial hydrogenic envelopes. The former contention is supjurted by the lack of association of SNI's with the spiral arms of (spiral) galaxie: within which they occur (Maza end van den Bergh 1976) and the occurrence of $\mathrm{SNl}$ in elliptical galaxies where the rate of massive star formation is v'ry small. The latter is consistent with the absence of hydrogenic lines in the SNI spectrum (unlike SNII) and the lack of a well defined "platrau" phase in the light curve (Falk and Arnett 1973; Weaver and Woosley 1980a). Most currently favored models are based upon white dwarfs accreting mass in cataclysmic variable systems (Wheeler and Hansen 1971; Whelan and Iben 1973; Mazurek 1973; Nomoto and Sugimoto 1977; Nomoto 1980ab, 1981, 1982ab, this volume; Taam 1980ab; Chevalier 1981; Weaver and Woosley 1980b; Weaver, Axelrod, and Woosley 1980, (WAW); Woosley, Weaver, and Taam 1980; Woosley and Weaver 1981; Woosley, Taam, and Weaver 1983,(WTW); Iben and Tutokov 1983) although a limited range of stellar masses between $8 \mathrm{M}_{\odot}$ and $10 \mathrm{M} \ominus$ may also contribute to the SNI rate (WAW) if such stars lose their hydrogen envelopes and irnite a degenerate thermonuclear runaway. 
The fate of the accreting white dwarf as well as the physical mecbanism for its explosion is critically dependent upon its mass, accretion rate, and the composition of the accreted matter (sce Iben and Tutokcv, 1983, for detailed discussion of various evolutionary scenarios expected to lead to SNI). Some combinations lead to common nova outbursts. If the thermonuclear model for novae as commonly formulated is correct (cf., Starrfieid, Spciks, and Truran 1974; Starrfield, Truran, and Sparks 1981; Mcdonald 1983), newly accreted material must mix with the carbonoxygen substrate during the inter-flash period. Since the nova itself does not appear likely to produce fresh carbon during its outburst, this implies that the white dwarf mass does not grow but may, in fact, shrink. Either way, the critical mass for a SNI is not attained. For some range of accretion rates and dwarf masses, though, the hydrogen shell flashes are expected to be weak (Fujimoto and Taam 1982; WTW; Nomoto this volume) and the mass of the dwarf does grow as a layer of degenerate helium accumulates between the hydrogen burning shell and the carbon-oxygen core (see Nomoto and Sugimoto 1977 for the interesting case of helium accretion onto a helium white dwarf). Providing that the accretion process is not truncated, the white dwarf has three possible fates: 1) off-center ignition with the accreted helium layer consumed in an outwardly propagating detonation wave and (most of) the carbon-oxygen core left behind as a lower mass white dwarf remnant; 2) off-center ignition with dual-detonation waves propagating inwards and outwards leading to the complete nuclear incineration and disruption of the star; or 3) a centrally ignited deflagration, with runaway occurring just short of the Chandrasekhar mass, that burns only the inner portion of the white dwarf but also leads to its complete disruption. We sitall briefly discuss representative models of each variety.

\subsection{Detonations and Deflagrations}

WTW have recently computed the complete evolution of three acereting white dwarfs, all of which experience "off-center" detonation (cases 1 and 2 above). The results should be representative of a broad range of aceretion rates and dwarf masses. Specifically examined were carbon-oxygen white dwarfs of mass $1.2,0.8$, and $0.5 \mathrm{M} \odot$ accreting helium (i.e., assuming hydrogen shell flashes to be weak or the donor star to be pure helium at rates of $10^{-8}, 2 \times 10^{-9}$, and $10^{-8} \mathrm{M} \mathrm{y}^{-1}$ respectively. We shall refer to these calculations as Models 1,2 , and 3 . All three models ignited helium burning under conditions that were extremely degenerate and, in all cases, this ignition was achieved prior to the star attaining the Chandrasekhar mass. The total masses of the white dwarfs at helium ignition were $1.43 \mathrm{M}_{\odot}, 1.34 \mathrm{M}_{\odot}$, and $1.12 \mathrm{M}_{\odot}$ respectively for Models 1, 2, and 3. Model 3 has been discussed previously (Weaver and Woosley 1980b; Woc ir, Weaver, and Taam 1980; WAW). The results of Model 2 are summarized in Figure 4.

The temperature and density as helium first begins to run away in Model 2 are $6.1 \times 10^{7}$ $\mathrm{K}$ and $7.5 \times 10^{7} \mathrm{~g} \mathrm{~cm}^{-3}$ respectively. Owing to the extreme degeneracy, the runaway proceeds 
all the way to the iron group, chiefly ${ }^{60} \mathrm{Ni}$ at this relatively low value of neutron excess, before substantial expansion occurs. The nuclear reactions release about $1.5 \times 10^{18} \mathrm{erg} \mathrm{g}^{-1}$ and raise the local pressure by about a factor of 5 . During the latter stages of the runaway the nuclear time scale becomes extremely short, much shorter than the sound crossing time for the burning region. The short burning time and large overpressure lead to expansion that, in the unburned material, is supersonic. Moving outwards the shock wave experiences a decreesing density gradient as well as a fuel (helium) that has a low ignition temperature. This allows the outward moving shock to propagate as a successful detonation wave, converting all but the outermost portions of the helium shell into iron. The inbound wave meets with less success. It must move up a density gradient and attempt to ignite a fuel (carbon) that has both a higher flash temperature and lower specific energy. As Figure 4 shows, the inward wave does not succeed in igniting the carbonoxygen core (as it does in Model 3; see Woosley, Weaver and Taam 1980), but simply dies out and becomes an acoustic wave which compresses and heats the core only slightly. Consequently, that layer which was helium is converted into iron and ejected with high velocity while most of the carbon-oxygen core is left behind as a bound reninant. Not all of the original white dwarf remains, however, only about $0.54 \mathrm{M}_{\odot}$ of the initial $0.8 \mathrm{M}_{\odot}$. This is because the total energy (internal minus gravitational) of the new, lower mass white dwarf is less than the total energy of the core of equivalent mass prior to removal of its outer layers and the difference is available for ejecting material. A total of $0.70 \mathrm{M} \odot$ is ejected by Model 2 with kinetic energy $1.15 \times 10^{{ }^{51}}$ erg. This ejected mass includes $0.26 \mathrm{M}_{\odot}$ of carbon and oxygen and $\sim 0.50 \mathrm{M}_{\odot}$ of ${ }^{60} \mathrm{Ni}$. Model 1 also leaves behind a white dwarf remnant, $1.16 \mathrm{M}_{\odot}$ in this case, and ejects a corresponaingly smaller amount of mass, $0.27 \mathrm{M}_{\odot}$, which is mostly ${ }^{60} \mathrm{~N}_{\mathrm{i}}$. The total kinetic energy is also much smaller, $3.8 \times 10^{60} \mathrm{erg}$. Model 3, as mentioned previously, leaves no remnant and generates an explosion of $2.2 \times 10^{51}$ erg. The light curves, spectra, and nucleosynthesis from these cxplosions are discussed in subsequent sections.

We have also recently studied a number of models characterized by centrally ignited deflagration. Niodel 4 is the end point of the evolution of a $2 \mathrm{M}_{\odot}$ helium core, which might typify a main sequence star of $\sim 9 \mathrm{M}_{\odot}$ that has lost its hydrogen envelope prior to the reduction of the helium core mass by convective dredge-up (WAW). This core spends 2.9 million years burning helium in a convective core of $0.73 \mathrm{M} \odot$. Typical conditions during this time (evaluated at $1 / 2$ helium depletion) are central temperature $1.6 \times 10^{8} \mathrm{~K}$, central density, $2.7 \times 10^{3} \mathrm{~g} \mathrm{~cm}^{-3}$, radius, $2.3 \times 10^{10} \mathrm{~cm}$, luminosity, $1.2 \times 10^{37} \mathrm{erg} \mathrm{s}^{-1}$, and effective emission temperature, $75,000 \mathrm{~K}$. During the next 300,000 years helium burns in a shell as the carbon-oxygen core grows to $1.1 \mathrm{M}_{\odot}$ (60\% carbon, 40\% oxygen). The envelope of the helium star also expands to $\sim 1.3 \times 10^{12} \mathrm{~cm}$ and the luminosity rises to $\sim 1.3 \times 10^{38} \mathrm{erg} \mathrm{s}^{-1}$. Neutrino losses become quite important during this stage, comparabie to the surface luminosity, and a central temperature inversion develops. 

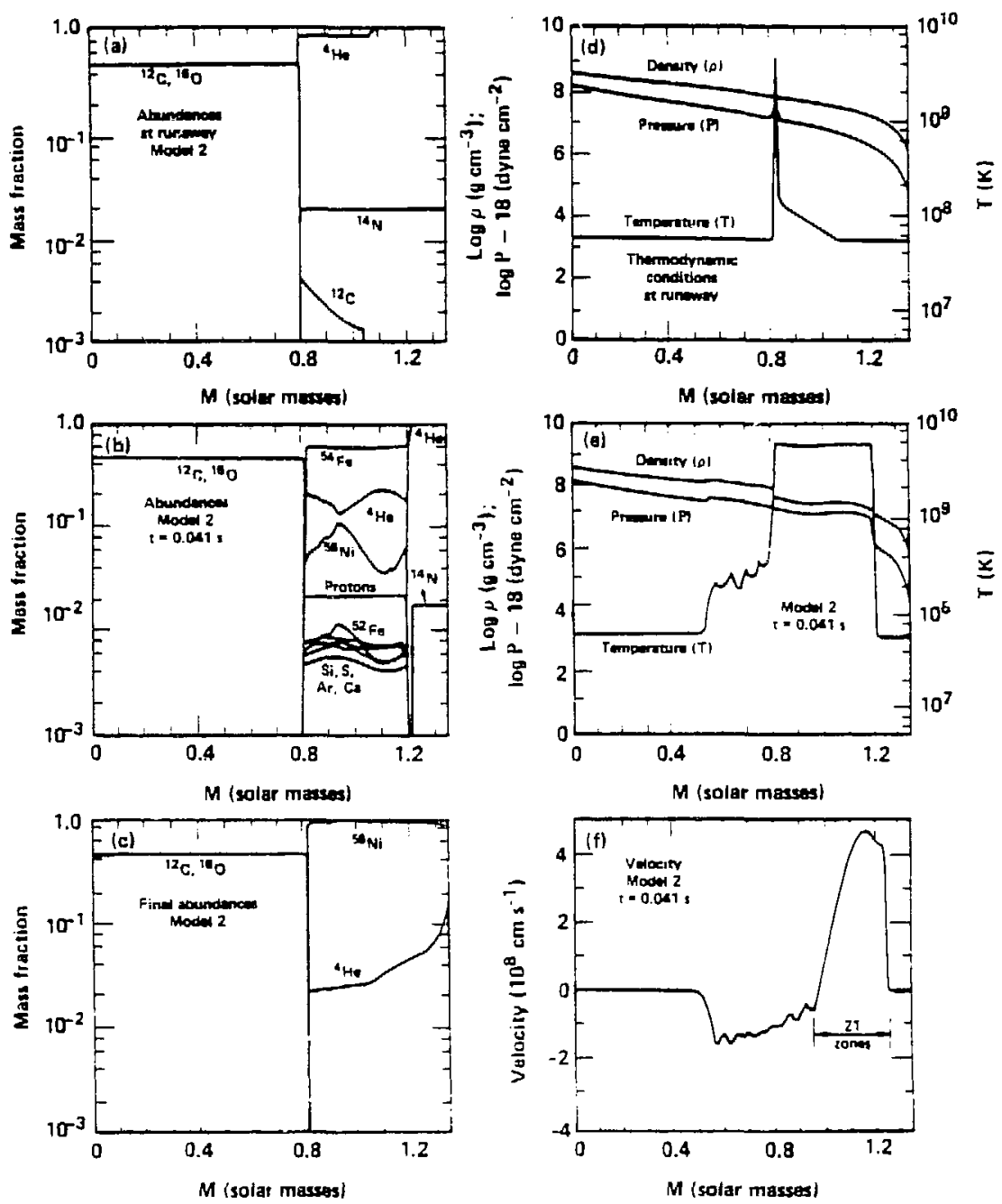

Figure 4. A sequence of figures showing the explosive evolution of "off-center" detonation Model 2 . The composition at the time of runaway, as defined by that time wher the temperature first reaches $5 \times 10^{\circ} \mathrm{K}$ at the base of the accreted layer (d) is shown in a. A small amount of helium has burned to ${ }^{12} \mathrm{C}$ before the runaway ensues. A short time later ( $41 \mathrm{~ms} ; b$, e, and $h$, a detonation wave has burned 0.4 $\mathrm{M}_{\odot}$ of the outlying material to iron group species. As expansion leads to cooling the ${ }^{54} \mathrm{Fe}$ shown in $b$ recombines with protons to produce ${ }^{6 s} \mathrm{Ni}$ as shown in $c$. The large overpressure and velocity in the outgoing stock and small overpressure and velocity of the inwardly proagating wave are seen in e and $f$. The carbon oxygen core never ignites. A portion is left behind as a white dwarf remnant of the supernova explosion. 
At this point, i.e., when the CO-core is $1.1 \mathrm{M}$, carben burning ignites non-degeneratoly, but off-center at $\sim 0.35 \mathrm{M}$. Typical conditions in the convective carbon burning shell are $7 \times 10^{8} \mathrm{~K}$ and $3 \times 10^{6} \mathrm{~g} \mathrm{~cm}^{-3}$, while the temperature at the center of the star is only $\sim 4 \times 10^{\mathrm{R}} \mathrm{K}$. Over the next 50,000 years the carbon burning shell moves inwards, eventually reaching the cutin of the star. During this time the boundary of the CO-core (i.e., the helium burning shell) mon $\therefore$ (nt to $1.22 \mathrm{M}_{\odot}$ and, over the next 15,000 years, to $1.3 \mathrm{M}$. At this time the inner $1.1 \mathrm{M}$ consists of a neon-magnesium core (60\% neon, $25 \%$ magnesium, and $15 \%$ oxygen) with the region 1.1 in 1.3 $\mathrm{M}$. composed of carbon and oxygen.

Here the helium shell burning became (perhaps unrealistically) violent. We purposefully maintained coarse zoning in the helium layer in order not to spend prohibitive amounts of computer time following thin shell instabilities. As the core grew from $1.3 \mathrm{M} \therefore$ to the Chandrasekluar value. successive belium shells burned one at a time as they added to the core. Owing to the degenerate conditions, high temperatures, up to 1.5 to $2.0 \times 10^{\circ} \mathrm{K}$, were attained $\mathrm{s}$, these zones experienced carbon, neon, and in some cases, even oxygen burning following their addition. This stage of the evolution is probably not realistic and should be computed with much finer zoning brfore it is taken seriously. The composition from 1.3 to $1.43 \mathrm{M} \odot$ must therefore be regarded, for the time being, as artificial.

Even so, the evolution of the central core should be correctly represented and, for purposes of exploring the generic class of deflagrating models, this should be adequate. It is, after all, the central density at runaway, and, to a lesser extent, the composition of the inner solar mass of material, that influences the outcome. The central dansity determines the mass and binding energy of the (nearly Chandrasekbar) core as wcll as the rate of electron capture oncc the explosion gets underway. The composition determines the specific encrgy released as burning proceeds to the iron group. Of course the exact composition of the outer layers will be important spectros'opically.

The conditions at runaway in Model 4, i.e., when citra! nucear energy generation first exceeded neutrino losses, were $2.9 \times 10^{8} \mathrm{~K}$ and $2.3 \times 10^{\circ} \mathrm{g} \mathrm{cm}^{-3}$. As the runaway developed, the central density reached a peak value of $3.7 \times 10^{9} \mathrm{~g} \mathrm{~cm}^{-3}$ before burning finally led to expansion. Burning in Model 4 was propagated as a convective deflagration, which is to say, ignition proceeded at a subsonic rate with overlying material ignited by the thermal transport of energy by convection from underlying material that was already burning. Numerical simulation of such a phenomenon using mixing length theory is highly uncertain at best, even in one dimension (see Muller and Arnett 1982 for a two-dimensional attempt). The temperature gradients are so extreme (super-adiabatic) that the convective velocity is near sonic. Peak temperature in the burning front is $\sim 7 \times 10^{9} \mathrm{~K}$ compared to only a few $\times 10^{8} \mathrm{~K}$ a short distance ahead of the front. Furthermore the time scale for nuclear burning is orders of magnitude shorter than the Courant crossing time for any reasonable zoning. Thus it is practically impossible to resolve the 
burning front on a numerical grid. This leads to a computational impasse which, if great care is not taken, may propagate the burning at an artificial velocity. This occurs, for example, if the luminosity from one zone to the rext is allowed to increase at an arbitrary rate set only by the (artificial) temperature gradient which may itself change on a very short time scale. As that gradient becomes large, the "convective luminosity" may become enormous heating the overlying zone rapidly to the flash point in a time short compared to what actual mixing could have achieved, even at the sound speed. After flashing, that zone can ignite the next one in a similar fashion and a chain reaction occurs that can erroneously propagate the burning at a supersonic veiocity even though the overpressure is not, in reality, enough to propagate a detonation wave.

We avoided this artificial propagation by restricting the rise in luminosity in a given time step to be no grater than the luminosity at the previous step multiplied by $\exp \left(\Delta t / \tau_{\text {conv }}\right)$ where $\tau_{\text {con }}$ is the convertive crossing time for the zone (thickness of the zone divided by the convective velocity which was, itself, limited to subsonic values). Obviously, this prescription is somewhat arbitrary and, for that reason, we regard the velocity at which the deflagration propagates as essentially a free parameter (limited, of course, by the speed of sound). Desides the central density at runaway, it is the most important parameter in the deflagrating modeis since it determines how much material will burn to iron and how quickly the star starts to expand and quenches the burning. For our calculations this critical parameter turned out to be roughly $1 / 3$ the speed of sound (or about $2000 \mathrm{~km} \mathrm{~s}^{-1}$ ). We note, in anticipation of subsequent discussions, that models which employ larger propagation velocities will synthesize more iron, develop greater expansion velocities, and experience less electron capture. The fnal composition and velocity for Model 4 are given in Figures 5 and 6 . The total kinetic energy at infinity was $3.5 \times 10^{50} \mathrm{erg}$.

We also prepared two additional models to simulate deflagration in accreting carbon-oxygen white dwarfs. Models 5 and 6 , composed initially of $50 \%$ carbon and $50 \%$ oxygen throughout their mass, were parametrized by their central densities at the time of runaway. We leave the task of relating these densities to actual evolutionary scenarios and, especially, accretion rates, to others (rf. Somoto 1982ab, Iben 1978ab, 1982: Taam 1980b; Iben and Tutukov 1983). In our calculations ignition was achieved by forming white dwarfs of nearly Chandrasekhar mass having initial central temperature $2 \times 10^{8} \mathrm{~K}$ and adding carbon and oxygen to their surfaces until they exploded. The preignition temperature should not be critical except as it partly deteinines the runaway density. In Model 5 explosion occurred at a peak central density $2.4 \times 10^{\circ} \mathrm{g} \mathrm{cm}^{-3}$ and in Model 6 explosion occurred at a peak density of $7.0 \times 10^{9} \mathrm{~g} \mathrm{~cm}^{-3}$. Final compositions and velocities are shown in Figures 5 and 6. The kinetic energies at infinity were $6.2 \times 10^{50}$ erg and $4.8 \times 10^{60}$ erg for Models 5 and 6 respectively.

In the final composition of all 3 deflagrating models one sees a tenth of a solar mass or so where burning at intermediate temperatures $\left(5 \times 10^{\circ} \geq T \gtrsim 2 \times 10^{\circ} \mathrm{K}\right)$ synthesizes elements (rom 


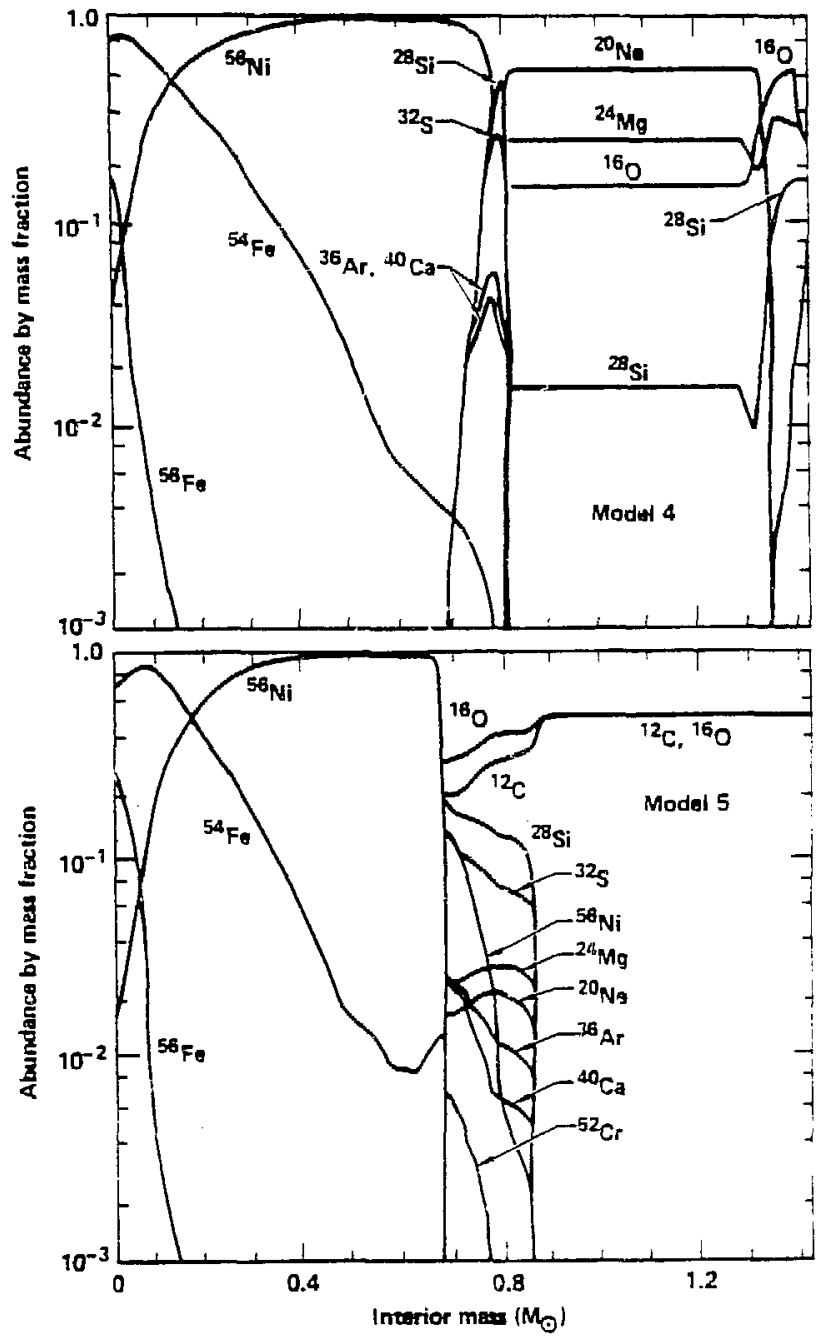

Figure 5. Final compositions of deflagrating Models 4 and 5 . A shell of the products of explosive oxygen turning surrounds the iron core in both cases. This is a result of the expansive quenching of the deflagration. Large quantities of ${ }^{54} \mathrm{Fe}$ are present in the cores of both models. The composition of the outer $0.15 \mathrm{M}_{\odot}$ of Model 4 may be artificial. 


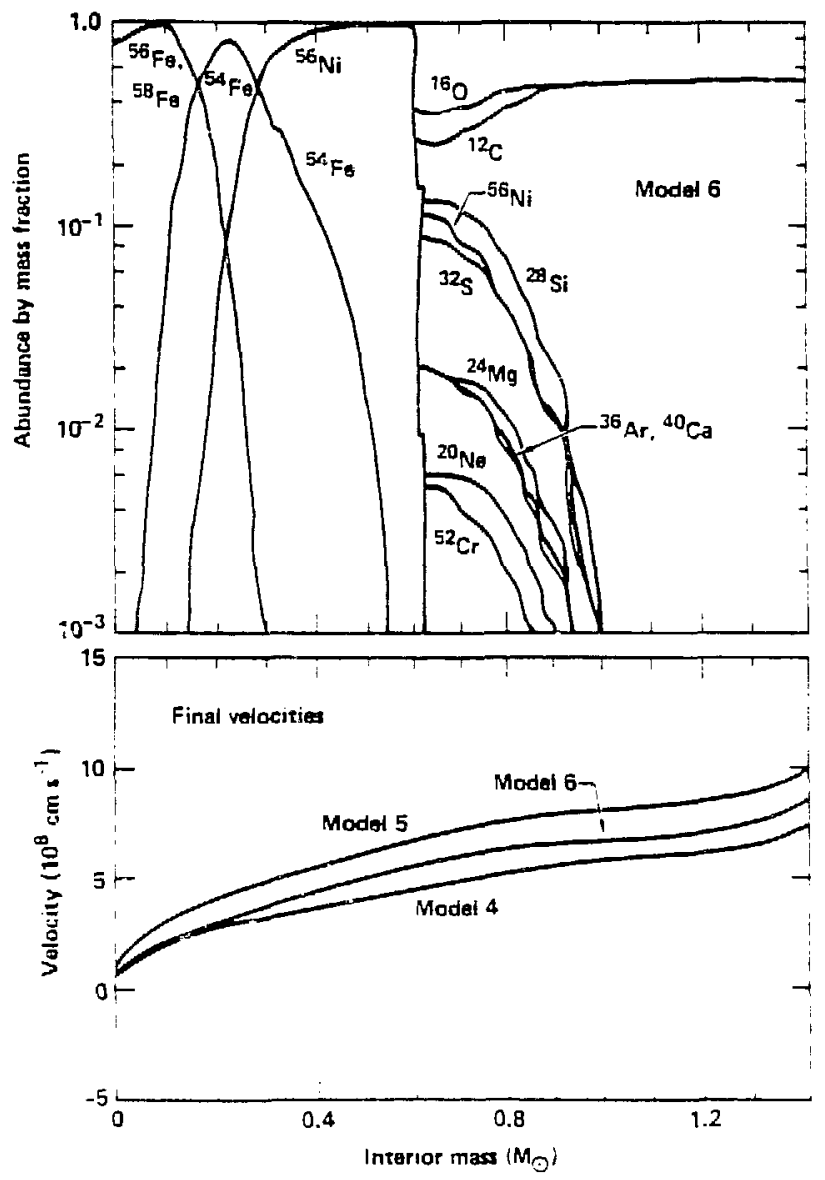

Figure 6. Final composition of deflagrating Model 6 and terminal velocities for all 3 deflagrating models. Owing to its large central density, Model 6 produces excessive quantities of neutron-rich isotopes. The velocity curves reflect the entral densities at runaway (Model 6 more tightly bound than Model 5) and the composition (less specific energy from neon burning in Model 4 than carbon and oxygen burning in Modeis 5 and 6 ). 
nend to calcium. This hurning transpires as the deflagration dies out. quenched by the expansion of the white dwarf. An interesting effece poorly modeled ir the present study is thr ming that may oereur for several seconds following the explusion owing to Raloigh-Taylor uns abling

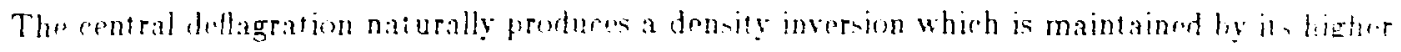

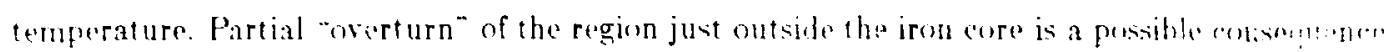

Another effect, seen in some calculations. but which we regard as "bogus" is racicial conver.tion occurring at very late times. The luminosity input by nickel decay is greatly sufer-lidingtun and. while the envelope remains optically thick (several days) and dominated by radiation pressure. it is formally unstable to overturning convection. Allowing this convertion en fromed at

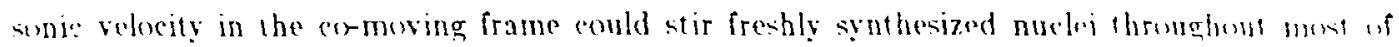
the exploding star. It is not proper. however. to apply traditional convection thewry in a merdium which is freely streaming and in which gravity has hecome a negligithe foree. The lummenity of a

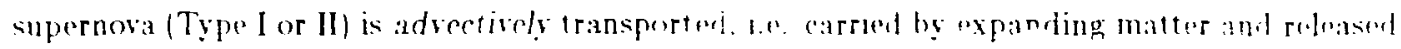
at the optically thin photosphere. The total energy in the radiation is at times later thar a few seconds, an inconsequential fraction of the streaming kinetice energy of the ejocta and. as surch. it can have negligible effect on its motion. We turned convection oft in our calculations at imm later than a few seconds.

\subsection{Light Curves}

It has long been realized that the light output of a Type I supernovae cannot be a comsurence simply of the high temperature and kinctic energy created during the explosion. Siomo late timm rencrgy input is required that is not degraded by adiahatic expansion. To ser this, one must only realize that the expanding white dwarfs of the previous section will not become optically thin until their radii hawe increased to roughly $10^{15} \mathrm{~cm}$. Thes estimate follows from an intial density of $\sim 10^{8}-10^{9} \mathrm{~g} \mathrm{~cm}^{-3}$ and an opaeity of a few tenths $\mathrm{em}^{2} \mathrm{~g}^{-1}$ (HAll). typical of the large ensemble of Doppler-hroadened lines in the expanding plasma. Since the initial radius was roughly a million times smaller than this, any initial internal energy depowited by the explikion is degraded to a trivial value. In Type Il supermue this problem is circumvented by starting with a configuration that already has a large radius, i.e. the low density envelope of a super-giant star.

The solution to this problem in SNI has also been realized for a long time, namely that the light curve of Type I's is a result of the delayed energy input by the decay of radioactive nuclei produced in the explosion (Borst 1950; Burbidge et al 195i), principally the nucleus ${ }^{50} \mathrm{Ni}$ $\left(\tau_{\frac{1}{2}}=6.1 d\right)$ and its daughter ${ }^{50} \mathrm{Co}\left(\tau_{\frac{1}{2}}=78.5 d\right)$ (Pankey 1962; Colgate and McKee 1967). It is also important to note that the decay of ${ }^{60} \mathrm{Co}$ proceeds $18 \%$ of the time though a mode that produces positrons. Thus even at late times when the supernova remnant becomes transparent to $\gamma$-radiation the kinetic energy of the positrons still deposits energy $(\sim 4 \%$ of the total decay 
energy) and ('an power the late time light curve (Arnett 1979; Axelrod 1980ab). It is also important that the time history of the emission will not be given simply by the decay rate of the radioactive nuclei hut will be modilated by both the efficiency of energy deposition by y-rays, a factor that is density dependent, and by a time dependent fraction of deposited energy that managed to mape withoul being degraded by adiabatic expinsion (WAW). The correct expression for the light outgut as is function of time is

$$
L(t)=I_{s 0} \dot{S}\left(t-\tau_{1}\right) f_{\text {dep }}\left(t-\tau_{1}\right) f_{e e c}(t)
$$

where $1 H_{i j}$ is the miss of freshiy synthesized ${ }^{60} \mathrm{Ni}, \mathrm{S}$ is the nuclear energy generation rate Erom ralinactive decay, $f_{d e p}$ is the fraction of the radioactive decay energy deposited in the

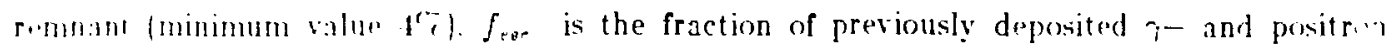
emergy thiat woids decompression to escape as optical (or near opticall radiation, and $\tau_{l}$ is the retarding lime to apporimaly rettect the mean lag time between energy deposition and escape (1) deempresinn) Analytic expressions that may be related to any supernova model are given for all of then quantitim hy WAW. It is interesting that the results are nost sensitive to i) the

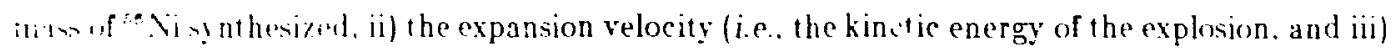
the lemity atreture of the expanding supernova. All these would be quite similar for exploting whit lwarle of nearly ('handrasekhar mass, which might explain why the class of Type I light curves is surbli a homog,neous sample.

The light curves for Models 1, 2 and 3 are given in Figure 7 . A detailed comparison of the light curve for Mfudel 3 and the observational data for supernova 1972e was given by WAIV and showed excellent : greement. Model 2 , with an appropriate but reasonable adjustment of supernova date and distance $11, \mathrm{YGC} 5253$. gives almost as good a fit. Light curves for the deflagrating models have not yet bren computed. but are expected to closely resemble Models 2 and 3 except that for Indel 4. the $2 \mathrm{M}$. helium star, there may be important modifications to its light curve brought axsut by the presence of -0.5 .11 of low densive helium surrounding the exploding enre. Model 1 is (ow) fast and cannot be made to fit the data at all well. The reasons for the dim, rapid display in Modul 1 are obriour. Tou little ${ }^{50} \mathrm{Ni}$ is synthesized and the ejecta move too fast, hence become thin to $;$-radiation too quicaly. Models 1 and 2 may, in fact, be even "faster" in their optical light curves than Figure 7 suggests. As we shall see in the next section, the emission from these explosions is dominated by lines of highly ionized iron that may, shortly after peak, emit principally in the ultraviolet. Thus there may be a substantial bolometric correction.

It is also important to recall that Models 1 and 2 leave white dwarf remnants. The calculations of ITW therefore suggest the existence of a class of SNI characterized by i) comparatively fast. dim light curves (hence rendering their detection less likely); ii) ultraviolet emission and "hot" 
spertra (see next section): and iii) white dwart stars present in their remnauts, perbaps wen wijt

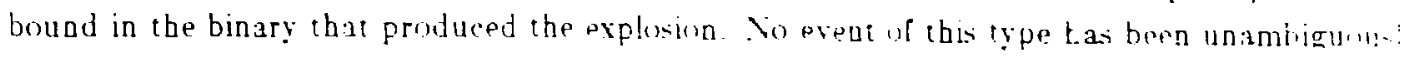

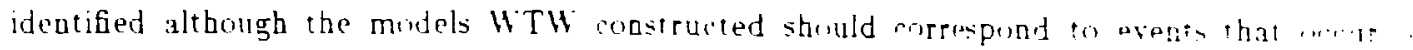
nature.

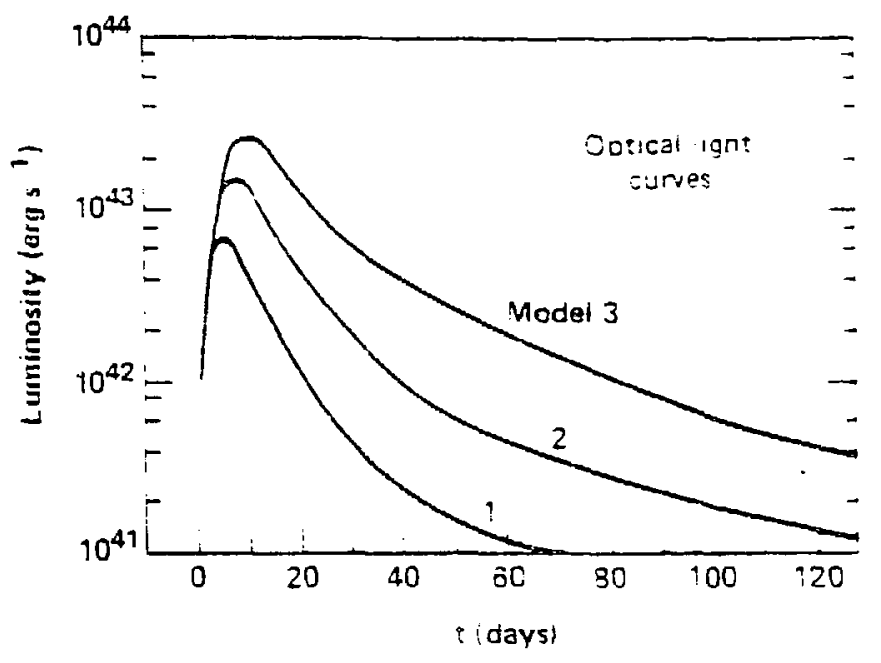

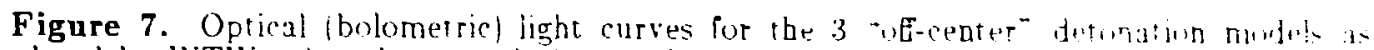
calculated by WTH asing the prescription of WAll. The ligbt curves are nust sensin t. thu mass of coxi ejected: the density of the expanding upernow: and he oradient in that dencit: Model 1 is an extremely "fast". dim Type I explosion because the mass if "Ni prodared is -mill and the expansion relocity grat. The factor of 4 rariation in peak luminnsity should ronghly

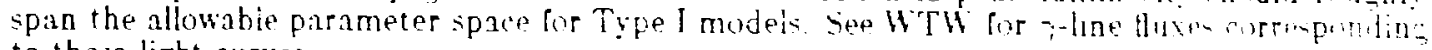
to these light curves.

\subsection{Type I Spectra}

As was first recognized by Meyernte $(1978$; 19003 il, the prosence of fist partirles from radioactive decay in the SXI plasma has important implications for the spectrum produed. In particular, ionization states may result which are far from LTE. Meyerott's work and the observation by Kirshner and Oke (1975) that the late time SNI spectrum bears a close resemblance to that of a pure, Doppler-broadened spectrum of Fe $I$ led Axelrod (1980ab) to undertake a numerical calculation of the spectra resulting from a uniform expanding shell of ${ }^{50} \mathrm{Ni}$. The numerical model self-c snsistently determined the temperature and ionization state of the nebula and accounted for a variety of spectral effects due to the rapid expansion of the nebula. Comparison of the results with the observed spectrum of SN1972e showed a close approximation was achieved if the ${ }^{50} \mathrm{Ni}$ 
had a maw in the ange 0.3 to 1.2 .11 . (depending on an unertain distance (o 2 (GC.5.5.3) and an

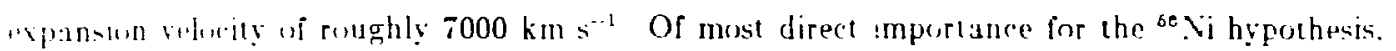

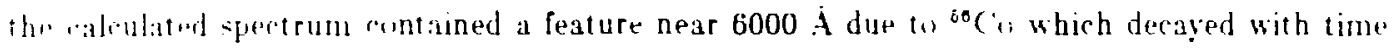
and a merespunting decaying feature was also present in the observations. This formed the first

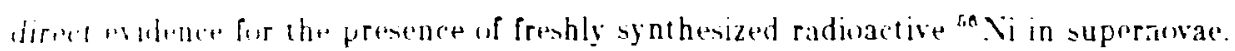

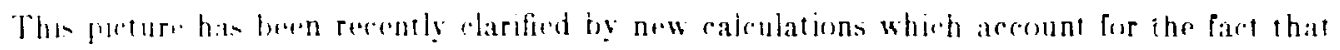
the s. Y upeitrim is strongly infuenced by the density profile of the ejecta. This roccurs because the junization balanere is very sensitive to density with both the average degree of ionizatiun and

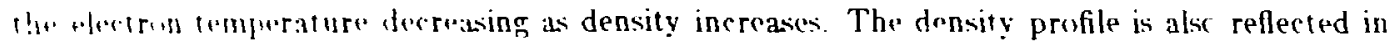
lhe yeretrum throlleh is effect on the transport of far ultraviolet radiation which results from rathatis. reombination. Wh have included these effects in ealculating the spectra form Models

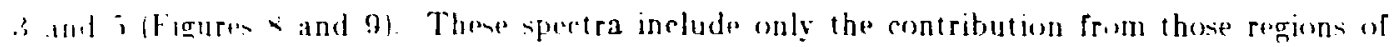

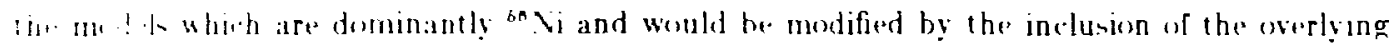
"amemplure". During ti.e nethlar phase. however. that at mesphere is expected to have only a

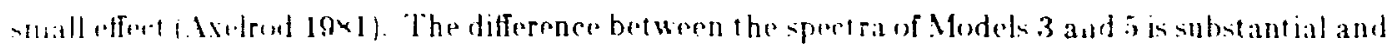

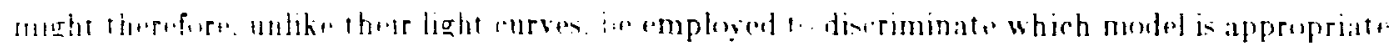

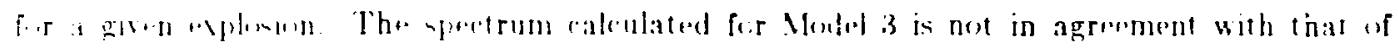

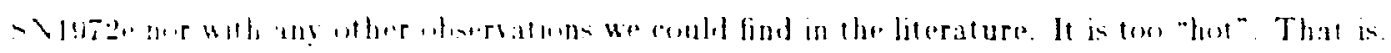

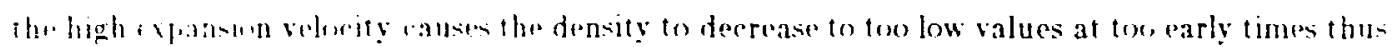

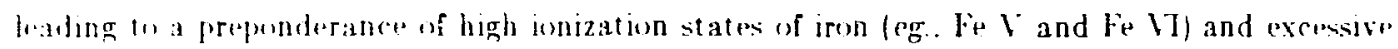

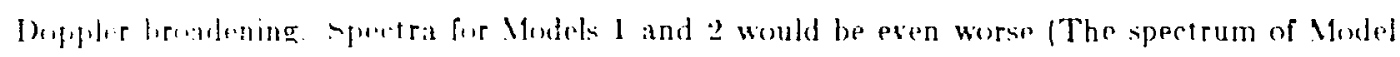

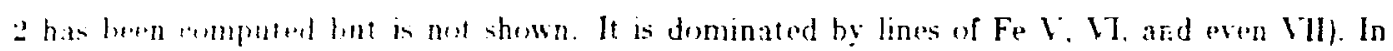

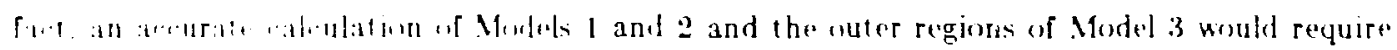
hishur trmperatures and innization tates than are currently arcessable by the code and would

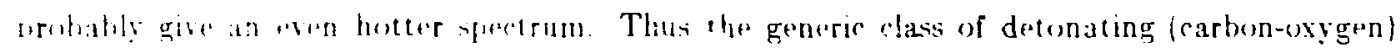

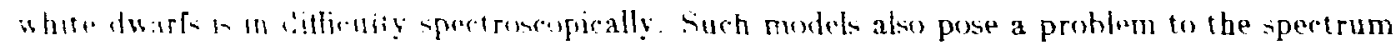

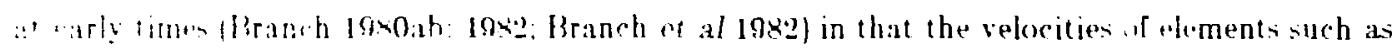

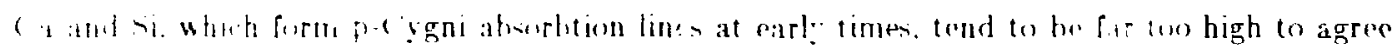

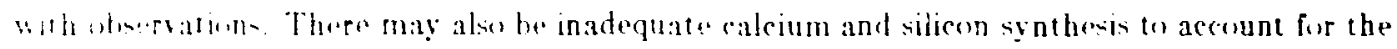

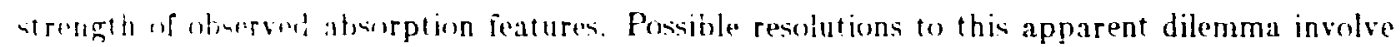
the two-dimensiunal gewnetry of the off center detonation, currently under study by Fryxell and Hoosley, or simply that this class of SNT models is relatively infrequent and our observational dat a base ton small.

On the other hand the computed spectrum for Model 5 is in good agreement with SNigize. in fact, given uncertainties in the atomic data, the agreement is excellent. Many details of the spectrum ire congruent in both theory and observation lending credence to the modeling process 

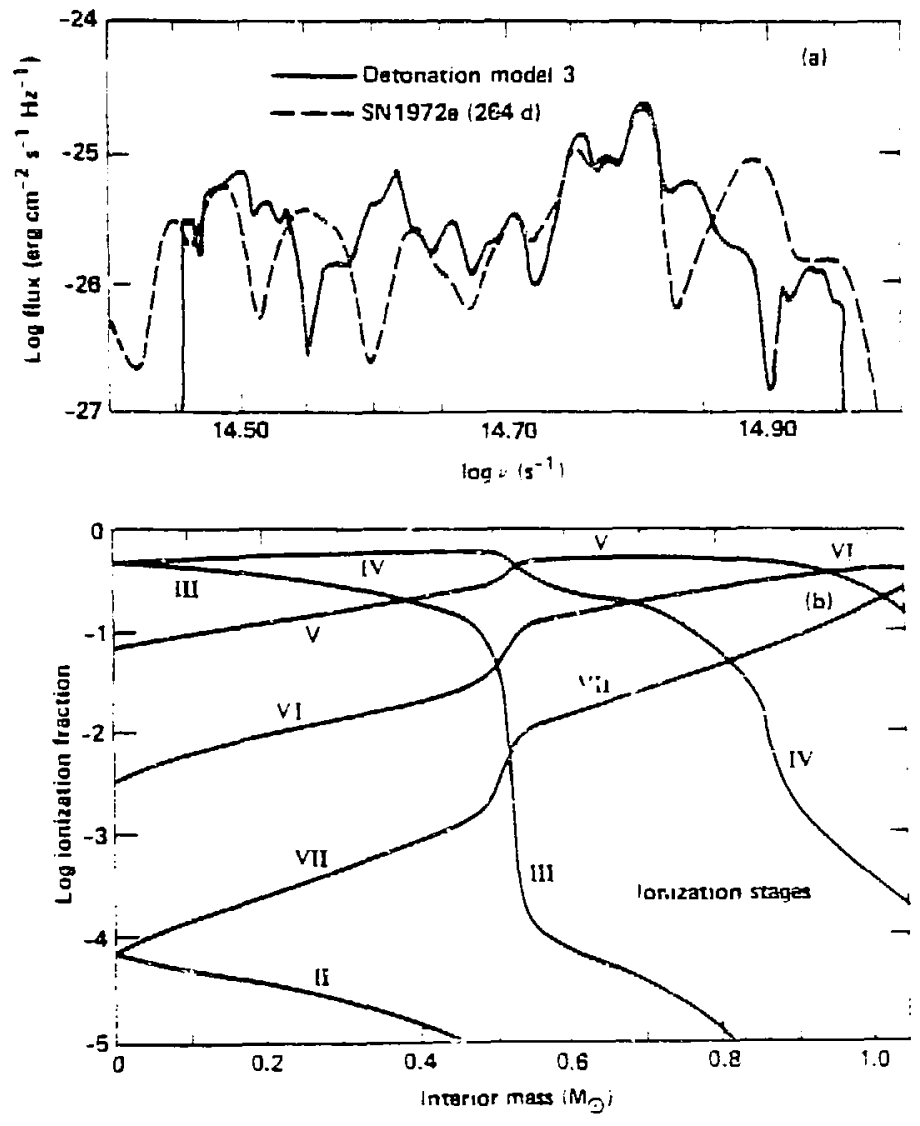

Figure 8. Optical spectrum of detonating Model 3 as compared to that observed (Kirshner et al 1973) from supernova 1972e 284 days after its explosion and the calculated ionization distribution of iron group species as a function of interior mass. Rapid expansion of the model results in a diminished abundance of free electrons and less efficient cooling. Thus highly ionized states of iron are abundant in the model that are not seen in the supernova. Line widths in tae model are also typically broader than observed. This is not a good ft (see also WAW and WTW). 

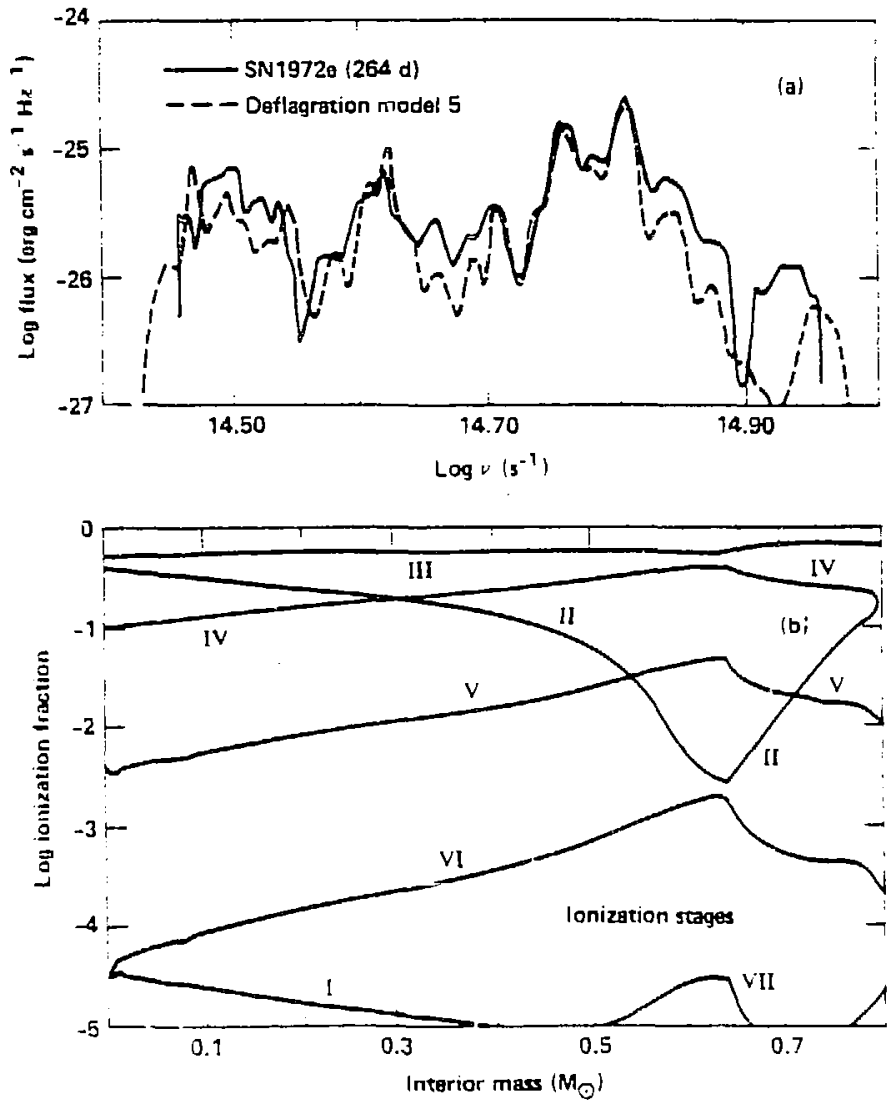

Figure 9. Optical spectrum of deflagrating Model 5 compared to supernova 1972e. The slower expension gives rise to narrower lines and less highly ionized states of iron and cobalt. This is a much better fit than Figure 8 . An absorption feature due to calcium is apparent in the observations at $\log \nu \approx 14.90$ which was not included in the model calculation. 
in gencral. The spectrum for Model 1 (not shown) has also been computed (without including the overlying atmosphere and is similar to that for Mudn 5, although a slightly "cooler". Medel 6 is expected to be similar. Moreover. the defagrating models offer antural site for the syntheis of silicon and calcium seren as absorption features in the observed spectrum (but not include. in the present spectral synthesis alculations). Model 5 makes - 0.02 M1 each of "si and 304 and - $0.003 \mathrm{Ms}$ each of ${ }^{36} \mathrm{Ar}$ and ${ }^{4 C} \mathrm{C} \mathrm{a}$. Model 1 makes $\sim 0.08 \mathrm{M}$ of ${ }^{28} \mathrm{Si}, \quad 0.02 \mathrm{M}$ of ${ }^{25} \mathrm{~S}$. $0.003 \mathrm{M} \approx$ of ${ }^{36} \mathrm{Ar}$, and $\sim 0.004 \mathrm{M}=$ of ${ }^{49} \mathrm{Ca}$. Cnfortunately the calcium in $\mathrm{Model} i \mathrm{is}$ moving at a maximum velocity of $8,000 \mathrm{~km} \mathrm{~s}^{-1}$, which is for slow according to Branch (1080:ab: $\left.10 \times 2\right)$. Indecel. all things considered, it would bo better if the deflagrating models had acepuirod groitler

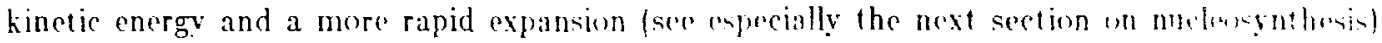
This might xecur if the deflagration wave has greater volocity. and as we argued that that veloxity is essentially a free parametre. such may well he the case. The deflagration of a helium duarf would also give a greater expansion velocity because of the greater specific energy arailab!: from burning helium to iron. There will, of course, always be some calcinun, as well as fracen of wh her heavy clements, present primordially in the outermont hycess which are ejected with the greatent velocity (roughly $11.006 \mathrm{~km} \mathrm{~s}^{-1}$ in slodel 5 ). Thus it is important to $\mathrm{knuw}$ quantitatively juit what fraction of the calcium abundanee is observed at what velncity. So far that deconvintion wif the observations has not been carried out. but the (simple one-dimensional) deflagraing models may be in some, hopefully slight, trouble here.

\subsection{Nucleosynthesis}

The chief nucleosynthetic pr tuct of these explosions is iron. principally the nucleus se fay sythesized, in part, although not exclusively in the doflagrating models, as its radionctive progenitor ${ }^{50} \mathrm{Ni}$. Owing to their rapid axpansion and comparatively low ignition density ulectron capture during the explosion of the detonating models is of no great consequence except for determining

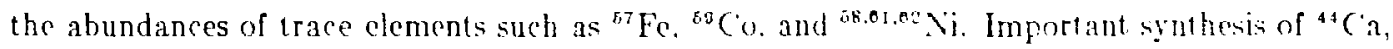
${ }^{\circ} \mathrm{Ni}$, and ${ }^{\circ} \mathrm{Zn}$ also occurs but these species are produced as radioartive progenitors $/{ }^{44} \mathrm{Ti},{ }^{\circ 0} \mathrm{Zn}$, and ${ }^{\text {a }}$ (e) in amounts that are relatively insensilive to the neutron oxess. Modelis 1. 2. and 3 ejert $0.21,0.50$, and $1.06 \mathrm{Mo}$ of iron group products respectively. Normalizirg to ${ }^{\text {sa }} \mathrm{Fe}$, the isotopes ${ }^{57} \mathrm{Fe},{ }^{50} \mathrm{Co},{ }^{00.01} \mathrm{Ni}$, and ${ }^{04} \mathrm{Zn}$ are produced in all three models within a factor of $30^{15}$ of their solar abundances. The specins: ${ }^{11} \mathrm{Ca}$ is underproduced by a factor ranging from 3 (Nodel 3 ) to 16 (Model 1), ${ }^{58} \mathrm{Ni}$ is underproduced by a factor of 2 to 6 , and ${ }^{02} \mathrm{Ni}$. by a factor of 3 to 20 . It is especially interesting that these models synthesize so little ${ }^{54} \mathrm{Fe}\left(<1^{\circ} 0\right.$ solar $)$. This is partly a consequence of the $\alpha$-rich Ireeze out (Arnett, Truran, and Woosiey 3371 ; Woosley. Arnett, and Clayton 1973) in which all ${ }^{54} \mathrm{Fe}$ is converted into nickel isotopes, and partly a result of the small neutron excess of these models. It is particularly interesting because no other models we have ever studied, including Type $\amalg$ supernova models, appear capable of making ${ }^{50} \mathrm{Fe}$ without ${ }^{64} \mathrm{Fe}$. 
A nucleosynthetic compc ant of this sort may be required in order to dilute the ${ }^{54} \mathrm{Fe}$-rich ejecta of other types of supernorae, especially the deflagrations we shall now discuss.

The nucleosynthesis from (defagrating) Models 4, 5, and 6 was given in Figures 5 and 6 . The finil electron mule number $\left(Y_{e}\right)$, is given for the inner core of all three models in Figure 10. 'The guantity $Y_{\text {; }}$ is rolated to the electron number density, $\mathrm{n}_{c}$. by $\mathrm{n}_{e}=\rho N_{A} y_{e}$ and the neutron excess, $\eta$. by $Y_{e}=1-2 \eta$. For nuclei having equal numbers of neutrons and protons, $r_{e}=1 / 2$. Owing to the high ignition density and comparatively slow expansion rate of these models. substantial electron capture occurs during the explosion leading to a decrease in $Y_{c}$ in thr. central core, so much so in fact that the dominant iron group nucleus at the center of the star whifts from first ${ }^{50} \mathrm{Ni}$ to ${ }^{54} \mathrm{Fe}$ to ${ }^{50} \mathrm{Fe}$ and, in the extreme case of Model 6, to ${ }^{88} \mathrm{Fe}$. Electron capture was calculated here using the older version of KEPLER that employs weak interaction rates from Mazurek (1973) and Hansen (1966) and the quasj-equilibrium network describud by Weaver, Zimmerman, and Woosley (1978). If anything, these weak rates should underestimate the actual electron capture fows. C cmpared to more realistic rates by Fuller, Fowler, and Newman (1982ab), key reactions, eg., ${ }^{80} \mathrm{Ni}\left(\mathrm{e}^{-}, \nu\right)^{50} \mathrm{Co}$, are generally slower by a factor of order 5 . Thus in reality one might expect the curves of Firure 10 to be shifted to slightly lower values of $Y_{\epsilon}$. ('There is some compensation sine the formation of a more neutron-rich daughter leads to a substantial reduction in the rate of neutronization. Also capture on free protons, a process that wais accurately modeled, is a niajor contributor to the decrease in $\mathrm{Y}_{\epsilon}$, especially for values near 0.51

Thr proncipal nuclensythetic creation of the deflagrating models is still iron group species but, owing wo the neutronization. there is considerable variation of the isotopic composition and none of it is very gond news. Model 4 ejects $0.53 \mathrm{M}$ of ${ }^{4} \mathrm{He}, 0.12 \mathrm{Mg}$ of ${ }^{10} \mathrm{O}, 0.30 \mathrm{M}$ of $={ }^{\circ} \mathrm{Ne}$,

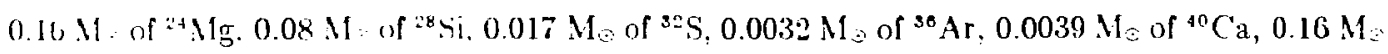
of " ${ }^{\circ} \mathrm{Fe}$, and $0.61 \mathrm{M}$. of ${ }^{50} \mathrm{Ni}$ which decays to ${ }^{50} \mathrm{Fe}$. The ${ }^{54} \mathrm{Fe}$ to ${ }^{58} \mathrm{Fe}$ ratio is an obvious problem. In thr sun this ratio is $0.06 \mathrm{l}$. In Model 4 it is 0.26 which means that supernovae like Model 4 whll haw been responsible for synthesizing no more than $225 \%$ of the iron in our Galaxy. Even ㄴ. the remainder of iron would have to be synthesized in objects: that make no ${ }^{54}$ Fe themselves, m.. the detomating models. Nor do Models 5 and 6 fare any better. Model 5 ejects 0.34 and 0.36 $\mathrm{M}$ : of unburned ${ }^{19} \mathrm{C}$ and ${ }^{10} \mathrm{O}$ (the near equality given by initial assumptions), $0.0037 \mathrm{M}_{\odot}$ of ${ }^{20} \mathrm{Ne}$, $0.0052 \mathrm{M} \odot$ of ${ }^{24} \mathrm{Mg}, 0.027 \mathrm{M}_{\odot}$ of ${ }^{28} \mathrm{Si}, 0.017 \mathrm{M}_{\odot}$ of ${ }^{32} \mathrm{~S}, 0.0035 \mathrm{M}_{\odot}$ of ${ }^{38} \mathrm{Ar}, 0.0033 \mathrm{M}_{\odot}$ of ${ }^{40} \mathrm{Ca}, 0.17$ $\mathrm{M}_{\supset}$ of ${ }^{54} \mathrm{Fe}$, and $0.49 \mathrm{M} \odot$ of ${ }^{50} \mathrm{Ni}$. This gives roughly the same ${ }^{54} \mathrm{Fe}$ constraint as Model 4 . Model 0 , because of its larger ignition density, faces even graver difficulties. Amounts of elemern lighter than iron are ejected that are comparable to Model 5 but, within the iron group, $0.15 \mathrm{M} \odot$ of ${ }^{54} \mathrm{Fe}$, $0.14 \mathrm{M} \odot$ of ${ }^{60} \mathrm{Fe}$ (with $0.31 \mathrm{M}_{\odot}$ made as ${ }^{60} \mathrm{Ni}$ and $0.13 \mathrm{M}_{\odot}$ made as ${ }^{58} \mathrm{Fe}$ itself), and $\sim 0.07 \mathrm{M}_{\odot}$ of ${ }^{58} \mathrm{Fe}$ are produced. This gives a ${ }^{54} \mathrm{Fe} /{ }^{50} \mathrm{Fe}$ ratio roughly 5 times that of the sun and a ${ }^{68} \mathrm{Fe} /{ }^{58} \mathrm{Fe}$ 


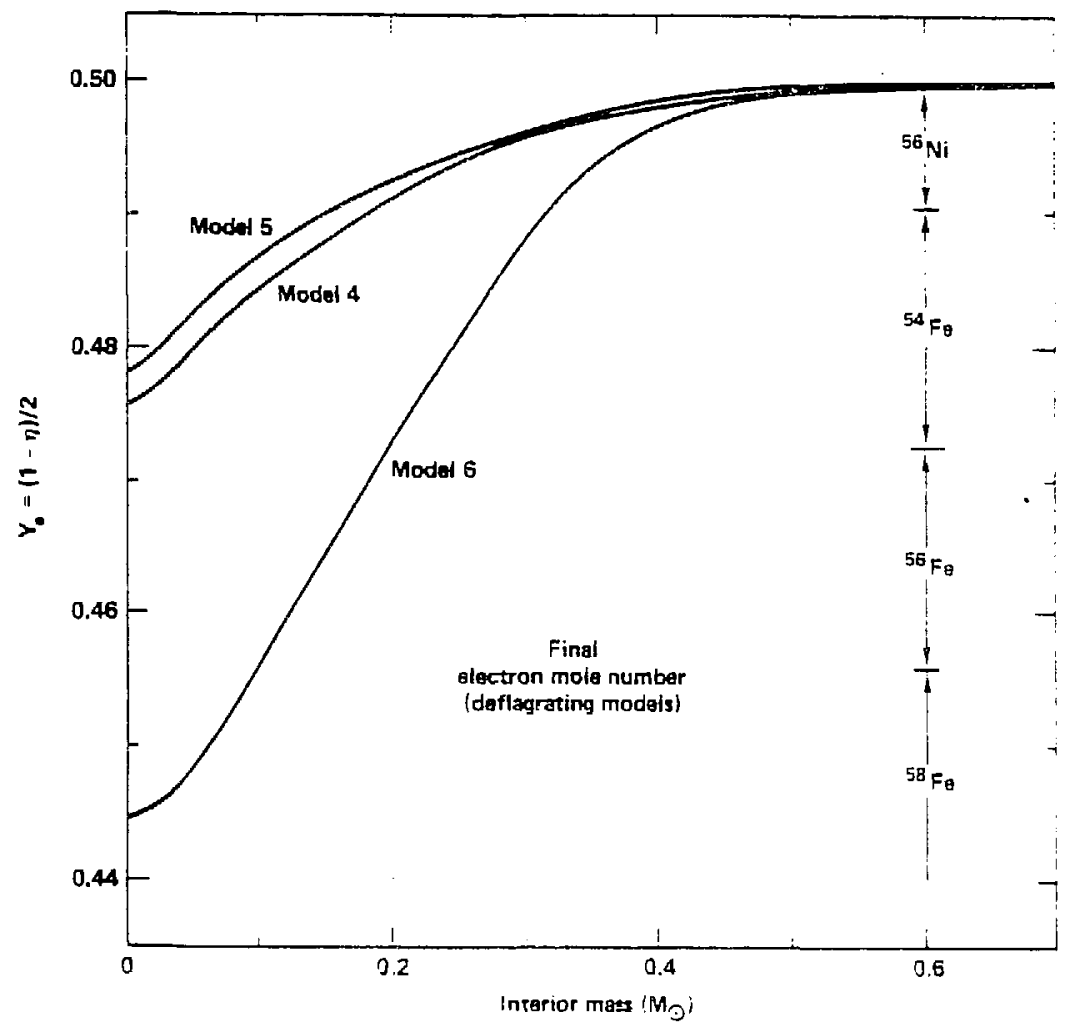

Figure 10. Distribution of the final electron mole number with interior mass in the 3 deflagrating models. The quantity $Y_{\text {a }}$ is initially 0.5 throughout the star (initial composition is of nuclei having $\mathrm{Z}=\mathrm{N}$ ) but is decreased by electron capture during the explosion. The dominant iron group isotope in nuclear statistical equilibrium at a given $Y_{\text {. }}$ is indicated by bandj. Large overproductions of ${ }^{54} \mathrm{Fe}$ and ${ }^{68} \mathrm{Fe}$ are a troubling aspect of deflagrating models. 
ratio roughly 40 times that of the sun! We conclude that deflagrating white dwarfs igniting at central densities $\gtrsim 2.5 \times 10^{\circ} \mathrm{g} \mathrm{cm}^{-3}$, i.e., the central density of Model 5 , must have been very rare in nature.

\subsection{Type I Summary}

We secm to have painted ourselves into a bit of a corner! All models studied here, with the possible exception of Model 1, apparently give reasonable light curves but the class of off-center detonations on carbort-oxygen cores produces unacceptable spectra (although quite reasonable nucleosynthesis) while the carbon-cxygen and neon deflagrations give unacceptable nucleosynthesis (although quite reasonable spectra). Something must be wrong. At this point we do not know the resolution of this dilemma. Obvious, but to us, presently unacceptable, solutions condd be i) SNI are not a major source of iron in nature; ii) our nuclear reaction rates and/or atomic dala are in much greater error than we helieve, or iii) the spectrum of SN1872e is not that of a typical SNI. More likely hypotleses we would suggest are i) the existence of another class of SNI models (helium dwarf deflatations.) having faster expansion rates and lower central ignitiun densities than our Models 1-6 but slower velocities than Models 1-3; ii) a low density "atmosphere" surrounding a detunating model which would "tamp" the high expansion velocities of the outer layers; iii) impriant 2 and 3 dimensional modifications to our current models, especially those involving off-cuter ignition, and/or iv) a preponderance of deflagrating models with central densities close to that of Model 5 but with more rapid velocities for the deflagration wave than we employed. OH,viously the search for the "correct" SNI model is still on.

One of the authors (SEV) gratefully acknowledges the hospitality and stimulating environment of the Erice conference on nucleosynthesis, especially conversations with D. Bond. D. Branch. ( . Chiosi, J. Galligher, and I. Iben. He also is grateful to the Lawrence Livermore National Laboratory for support and computer time over the last 14 months while the work described here was carried out. This work has been supported by the National Science Foundation (AST-81-08509) and under the auspices of the I'.S. Department of Energy by the Lawrence Livermore National Laboratory under Contract W-740s-Eng-48. 


\section{DISCUSSION}

Edmunds: Can you predict what will happen to nucleosynthesis in the new $25 M_{\odot}$ star?

Woosley: Qualitatively the results should resemble what was calculated for the old $25 \mathrm{M}_{\odot}$ star. In detail, the new model may give better production of the silicon to calcium group because the core of the new star is surrounded by material with a shallower density gradient. It is our hope that material which once was a part of the old iron core will now experience explosive oxygen burning with an enhanced production of intermediate mass elements. This remains to be seen. Also you may note (Fig. 2) that the helium core is a little smaller (by about I $\mathbf{M}_{\odot}$ ) and carbon abundance somewhat larger (by about a factor of 2) than reported by Woosley and Weaver $1982 \mathrm{~b}$.

Thielemann: The peak in the abundance curve you and Weaver published (Woosley and Weaver 1982b) contains carbon burning products while the minimum contains oxygen burning products. The new ${ }^{12} \mathrm{C}(\alpha . \gamma)^{10} \mathrm{O}$ rate (Kettner et al 1982), which is enhanced by a factor of 3 to 5, will lave less carbon and more oxygen after helium burning. Since this changes the fuels for subscauent burning stages will your yield curve be flattened (i.e., inproved) by using the new rate?

Woosley: As Willy Fowler has long reminded us, we must get the reaction rates right before we can trust the nucleosinthesis calculations. However, I do not expect the yields of intermediate mass nuclei to be grossly affected (I differ with you and Arnett here). These nuclei are produced by explosive oxygen burning and, as Arnett, Clayton and I discussed many years ago (1973), it is the peak temperature in the explosion which most senitively determines its yield. That is, a carbon composition exposed to the same peak temperature (above $T_{0} \approx 3$ ) as an oxygen composition will give the same nuclensynthesis. Memory of the initial composition is lost. The aplosion temperature is set by the pre-explosive density gradient and by the explosion energy of the supernova, both of which are relatively insensitive to the rate for ${ }^{12} \mathrm{C}(\alpha, \gamma)^{10} \mathrm{O}$. The ratio of carbon to oxygen in the ejecta may, of course, change greatly.

Trautvetter: Since you mentioned ${ }^{20} \mathrm{Al}$ as an important candidate for $\gamma$-line astronomy, I would like to comment that our group has recently measured the cross section for ${ }^{20} \mathrm{Al}(\mathrm{p}, \gamma)^{27} \mathrm{Si}$ which could eventually destroy Ireshly produced ${ }^{2 n} \mathrm{Al}$. We lound, in the energy range $0.27 \lesssim \mathrm{E}_{p}$ $\leqslant 1.8 \mathrm{MeV}$, five resonances which are all very weak indicating that ${ }^{20} \mathrm{Al}$ can survive H-burning.

Woosley: That is very interesting, especially for ${ }^{20} \mathrm{Al}$ production in novae. Having done the difficult task of preparing an ${ }^{20} \mathrm{Al}$ target you should now measure the $(\mathrm{n}, \mathrm{p})$ cross section 
since that is the principal destruction mechanism in explosive neon burning which dominates ${ }^{20} \mathrm{Al}$ production in supernovae (Woosley and Weaver 1980).

Renzini: I have 3 short questions: 1) What was the surface $\mathrm{N} / \mathrm{O}$ ratio in the $25 \mathrm{M} \leftrightharpoons \mathrm{star}$ at the time of its explosion? 2) Where is the ${ }^{17} \mathrm{O}$ produced? and 3) Has neon production in the new model changed substantially? The neon production you obtained in earlier models was quite different from Arnett (1978).

Woosley: The surface $\mathrm{N} / \mathrm{O}$ ratio is close to unity (actually 4:5; see Fig. 2). The species ${ }^{17} \mathrm{O}$ is produced by hydrogen shell burning by the CNO tri-cycle. Neon production has not changed substantially. It is still large.

Renzini: Iben and Truran (1978) have shown that m.ssive AGB ctars (if they exist) nay make large amounts of ${ }^{22} \mathrm{Ne}$ which, in this case, is mostly primary. Anomalies of ${ }^{22}$. Ye could then be incorporated into grains forming around such $A G B$ stars.

Woosley: Perhaps, but it might be difficult to incorporate 22Ne, a noble gas, into atellar grains. There exist in the solar system components of neon in meteorites (Ne E) that are almost pure ${ }^{22} \mathrm{Ne}$. $\mathrm{A}^{22} \mathrm{Na}$ carrier which decays after chemical incorporation seems more likely.

Bond: Could you comment on your work with Bodenheimer concerning rotation and oxygen burning driven ejection in massive stars? This is related to the question of how to get Cygnus $\mathrm{X}-1$ black holes with explosions.

Woosley: That work is now published (Bodenheimer and Woosley 1083). The major uncertainty is our treatment of the inner boundry condition which must act, at least partly, to inhibit the accretion flow. We are encouraged that supernova remnants have been observed that do exhibit toroidal symmetry.

Nomoto: What is the propagation velocity of the deflagration wave in your Type I models?

Woosley: It varies, but typically $\sim 2000 \mathrm{~km} \mathrm{~s}^{-1}$, or about $1 / 3$ the speed of sound.

Nomoto: I have also calculated the model of a single detonation leaving a white dwarf remnant behind. Such events occur in a relatively small region of parameter space (i.e., accretion rate vs white dwarf mass) and might therefore be expected to be relativiely rare. 
Woosley: Your calculations may have underestimated the probability of leaving a white dwarf bchind because they do not account for the transfer, by hydrodynamical means, of energy in the central portions of the white dwarf to the kinetic energy of ejecta. The total energy of the white dwarf core stripped of its helium (now iron) envelope may be zero, or even positive, and still leave a rempant because excess energy goes into accelerating a portion of the outer layers to high velocity. This happened, in fact, in Model 2 which I believe according to your phase diagram would not have been expected to leave a remnant. Still the parameter space may be restricted. I agree.

Braun: If it is indeed the case that the SNI light curve shape is relatively insensitive to the details of the model, we may have to wait for a more complete sample of SNI spectra before demanding that there be a unique class of progenitors.

Woosley: I certainly agree we need more spectral data, particularly at late times, but Dave Branch tells me that many spectra have been taken near peak light and they all fit one another like gloves. Perhaps it is only the late time spectrum (which is all that Axelrod is presently capable of modeling) that is so discriminatory.

Rayet: What would be the physical reason why Nomoto and Hillebrandt claim the possibility of an r-process site in their $9.6 \mathrm{M}_{\odot}$ explosion while you do not mention it for your $10 \mathrm{M}_{\odot}$ model? Is it related to the steep entropy increase during explosicn quoted by Nomoto?

Woosley: Evolutionary endpoints vary rapidly in the mass range 9 to $10 \mathrm{M}_{\odot}$. Our $10 \mathrm{M}_{\odot}$ star underwent an ordinary core collapse following silicon core depletion. Nomoto's $\mathbf{9 . 6} \mathrm{M}_{\odot}$ model, if my memory serves me correctly, collapsed on electron rapture prior to oxygen depletion. The smaller core mass and energy input from nuclear burning during the collapse combined to produce an energatic explosion. I am not surprised that they get production of large amounts of neutronrich isotopes in such a powerful explosion. In fact, they may find it far too much of a good thing. That is. overproduction of neutron-rich isotopes may be a problem. 


\section{LIST OF REFERENCES}

Arnett, W. D. 1979, Ap. J. Lettr., 230, L37.

-. 1980, Ann. N. Y. Acad. Sci, 336, 366.

Arnett, W. D., Truran, J. W., and Woosley, S. E. 1971, Ap. J., 165, 87.

Appenzeller, I., and Fricke, K. J. 1972, Astron. and Ap., 21, 285.

Appenzeller, I., and Tscharnuter, W. 1973, Astron. and Ap., 25, 125.

Axelrod, T. S. 1980a, PhD thesis, Univ. California at Santa Cruz and Lawrence Livermore Laboratory Report UCRL-52994.

- - 1880b, in Type I Supernovae, ed. J. C. Wheeler (Austin: University of Texas), p. 80.

-.. 1981. Lawrence Livermore Laboratory Report UCRL-86850 and in preparation for Ap. $J$

Barkat, Z., Rakary, G., and Sack, N. 1967, Phys. Rev. Lettr., 18, 379.

Besisl. M. S., and Vorris, J. 1981, LAU Colloq. No. 86: Astrophysical Parameters for Globular

Clusters, ed. A. Ci. D. Philip and D. S. Hayes, (L. Davis Press; Schenectady), 137.

Bodenheimer. P. B.. and Woosley, S. E. 1983, Ap. J., 269, 281.

Borst, I. B. 19:50, 'This. Rev., 78, 807.

Bund. II. E. 1081 . 1p. J., 248, 606.

Bond. J. R.. Arnett, W. D.. and Carr, B. J. 1982, in Supernovae: A Survey of Current Researih, rd. M1. J. Rees and R. J. Stoneham, (D. Reidel: Dordrecht).

....., 1983, Ap. J., in press.

Bowers, R. L.. and Wilson, J. R. 1982a, Ap. J. Suppl., 50, 115.

....... 198:2b, Ap. J., 263, 366.

Branch. D. 1980a, in Supernovae Spectra, ed. R. Meyerott and G. H. Gillespie, (Am. Inst. Phys.: Lew York). Proc. No. 63, p. 39.

- 1980b, in Type I Supernovae, ed. J. C. Wheeler (Austin: University of Texas), p 66.

- 1982. Iroc. XI Texas Symp. on Relativistic Ap., to be published by Ann. N. Y. Acad. Si.i.

Branch. D.. Buta, R. Falk, S. W., McCall, M. L., Sutherland, P. G., Uomoto, A., Wheeler, J. C'.. and Wills, B. J. 1982, Ap. J. Lettr., 252, L61.

Brown, (. F., Bethe. II. A., and Baym, G. 1982. Nuclear Physics, A375, 181.

Burbidge, E. M., Burbidge, G. R., Fowler, W. A., and Hoyle, F. 1957, Rev, Mod. Phys., 29, i) 17 .

Butler, D., Dickens, R. J., and Epps, E. 1978, Ap. J., 225, 148.

Cameron, A. G. W. 1973, Spac. Sci. Rev., 15, 121.

Chevalier, R. A. 1981, Ap. J., 246, 267

Colgate, S. A., and McKee, C. 1969, Ap. J., 157, 623.

Colgate, S. A., and Petschek, A. 1979, Ap. J. Lettr., 236, L115.

Colgate, S. A., Petsehek, A. G., and Kriese, J. T. 1980, Ap. J. Lettr., 237, L61.

Cox, A. N., and Stewart, J. N. 1970a, Ap. J. Suppl., 19, 243. 
1970b, Ap. J. Suppl., 10, 261.

Dicke, R. H., and Peebies, P. J. E. 1968, Ap. J., 154, 891.

Epstein, R. 1979, MARAS, 188, 305.

Ezer, D., and Cameron, A. G. W. 1871, Ap. and Spac. Sci., 14, 398.

Falk, S. W., and Arnett, W. D. 1973, Ap. J. Lettr., 180, L65.

Fowler, W. A. 1966, in High Energy Astrophysics, ed. L. Gratton, (Academic Press: Sew York), 313.

Fricke, K. J. 1973, Ap. J., 183, 941.

-. 1974, Ap. J., $189,535$.

Fujimoto, M. Y., and Taam. R. E. 1982, Ap. J., 260, 249.

Fuller, G. M., Fowler, W. A., and Newman, M. J. 1982a, Ap. J. Suppl., 48, 279.

1082b, Ap. J., 252, 715.

Fuller, G. M., Woosley, S. E., and Weaver. T. A. 1982, Bull. Am. Astron. Soc. 14, 957.

- 1983, in preparation for Ap. J.

Hansen, C. J. 1966, PhD Thesis, Yale Lniversity.

Hillebrandt, W, 1982a, Astron. and Ap., 110, L.3.

- - 1982b, in Supernovae: A Surver of Ciurrent Research, ed. M. J. Ress and R. J. Stonehan, (D. Reide!: Dordrecht).

-- -. 1983, as presented at X7 Texas Sỵmposium on Relativistic Astrophysies, to be published by Ann. N. Y. Acad. Sci, preprint.

Iben, I. 1963. Ap. J., 138, 1090.

- $1878 \mathrm{a}$, Ap. J., 219, 213.

- $1978 \mathrm{~b}$, Ap. J., 226, 996.

-... 1982, Af. J., 253, 248.

Iben, I., and Tutukov, S. 1983, preprint. Lniv. of Illinois.

Kirshner, R. P., and Oke, J. B. 1875, Ap. J., 200, 574.

Kirshner, R. P., Oke, J. B., Penston. M. V., and Searle. L. 1973, Ap. J., 185. 303.

Maza, J., and van den Bergh, S. 1976. Ap. J., 204, 519.

Mazurek, T. J. 1973, Ap. and Spac. Sci.. 23, 365.

M.Donald, J. 1983, Ap. J.. 267, 732.

Meyerott, R. E. 1978, Ap. J., 221.975.

--. 1980a, in Supernovae Spectra, ed. R. Meyerott and G. H. Gillespie, (Am. Inst. Phys.: New York), Proc. No. 63, p. 49.

- 1980b, in Type I Supernovae, ed. J. C. Wheeler, (University of Texas), p. 72.

Miyaji, S., Nomoto, K., Yokoi, K., and Sugimoto, D. 1980, Pub. Astron. Soc. Japan, 32, 303.

Muller E., and Arnett, W. D. 1982, Ap. J. Lettr., 261, L109.

Nomoto, K. 1980a, in Type I Supernovae, ed. J. C. Wheeler (Austin: University of 'Texas), p. 164.

ㄱ... 1980b, Spac. Sci. Rev, 27, 563. 
-. 1981, in LAU Symposium 83, Fundamental Problems in the Theory of Stellar Evolution, ed. D. Sugimoto, D. Q. Lamb, and D. N. Sckramm (Dordrecht: Reidel), p. 295.

-. 1982a, Ap. J., 253, 798.

- 1882 b, Ap. J., 257, 780.

- -. 1983, Max-Planck Inst. fur Physik und Astrophysik Preprint MPA72.

Nomoto, K., and Sugimoto, D. 1977, Publ. Astron. Soc. Japan, 29, 765.

Norgaard, H., and Fricke, K. J. 1976, Astron. and Ap., 49, 337.

Ober, W. 1981, Ph D Thesis, Universitat zu Gottingen.

Ober, W., El Eid, W., and Fricke, K. J. 1983, Astron. and Ap., 119, 61.

Palla, F., Salpeter, E. E., and Stahler, S. W. 1983, Ap. J., $271,632$.

Pankey, T. 1962, PhD Thesis, Howard University. Diss. Abstr., 23, 4.

Papaloizou, J. C. B. 1973, Mon. Not. R.A.S., 162, 169.

Sanders, R. H. 1970, Ap. J., 162, 781.

Silk, J. 1977, Ap. J.,211, 638.

Smarr, L., Wilson, J. R., Barton, R., and Bcwers, R. 1981, Ap. J., 246, 515.

Sneden, C., Lambert, D. L., and Whitaker, R. W. 1979, Ap. J., 234, 964.

Starrfield, S. G.. Sparks, W. M., and Truran, J. W. 1874, Ap. J. Suppl., 28, 247.

Starrfield S. G., Truran, J. W., and Sparks W. M. 1981, Ap. J. Lettr., 243, L27.

Stothers, R., and Simon, 1970, Ap. J., 160, 1019.

Sugimoto, D., and Nomoto, K. 1980, Spac. Sci. Rev., 25, 155.

Taam. R. E. 1980a, Ap. J., 237, 142.

..... 1980b, Ap. J., $242,749$.

Talbot, R. J. 1971, Ap. J., 165, 121.

Trimble, V. 1982, Rev. Mod. Phys., 54, 1183.

Wallace, R. K., and Woosley, S. E. 1981, Ap. J. Suppl., 45, 389.

Wallace, R. K.. Woosley, S. E., and Weaver, T. A. 1982, Ap. J., 258, 696.

Weaver. T. A., Zimmerman, G. B., and Woosley, S. E. 1978, Ap J., 225, 1021.

Weaver, T. A., and Woosley, S. E. 1979, Bull. Am. Astron. Soc., 11, 727.

-.-. 1980a, Ann. N. Y. Acad. Sci., 336, 335.

-. 18:0b), in Supernovae Spectra, ed. R. Meyerott and G. H. Cillespie, (Amer. Inst. Phys: New lork), Proceedings No. 63, p. 15.

Weaver, T. A., Axelrod, T. S., and Woosley, S. E. 1980, in Type I Supernovae, ed. J. C. Wheeler (Austin: University of Texas), p 113 (WAW).

Weaver, T. A., Woosley, S. E., and Fuller, G. M. 1982, Bull. Am. Astron. Soc, 14, 957.

- 1983, in Numerical Astrophysics: In Honor of J. R. Wilson, ed. R. Bowers et al, (Science Books Intern'l: Portola CA), in press (WWF).

Wheeler, J. C. 1881, Rept. Prog. Phys.,44, 85.

Whwler, J. C., and Hansen, C. 1971, Ap. and Spac. Sci., 11, 373.

Whelan, J., and lben, I. 1073, Ap. J., 186, 1007. 
Wilson, J. R. 1983, in Numerical Astrophysics: In Honor of J. R. Wilson, ed. R. Bowers et al, (Science Books Intern'l: Portola CA), in press.

Woosley, S. E. and Weaver, T. A. 1980, Ap. J., 238, 1017. --.. 1981, Ann. N. Y. Acad. Sci., 375, 357.

-2. 1982a, in Supernovae: A Survey of Current Research, ed. M. J. Rees and R J. Stoneham, (D. Reidel: Dordrecht), p. 79.

- 1982b, in Essays on Nuclear Astrophysics, ed. C. A. Barnes, D. D. Clayton, anc D. N. Schramm, (Cambridge Univ. Press), p. 377.

Woosley, S. E., Weaver, T. A., and Taam, R. E. 1980, in Type I Supernovae, ed. J. C. Wheeler (Austin: University of Texas), p. 96.

Woosley, S. E., Arnett, W. D., and Clayton, D. D. 1973, Ap. J. Suppl., 231, 26.

Woosley, S. E., Taam, R. E., and Weaver, T. A. 1883, in preparation for Ap. J. (WTW). 


\section{DISCLAIMER}

This ducument was prepared as an account of work sponsored by an agency of the Unilet States Government. Neither the Unjted States Government nor the tniversity of Califomia nor any of their employees, makes any warranty, express or implied, or assumes any legal hiability or responsibility for the accuracy, completeness, or usefulriess of any information, apparatus, product, or process disclosed, or represents that its use would not infringe privately owned rights. Asference herein to any specific commercial products, process, or service by trade name, trademark, manufacturer, or otherwise, does nol necessarily constitute or imply its endorsement, recommendation, or faroring by the United States Government or the l'niversity of California. The riews and opinions of authors expressed herein do not necessarily state or reflect those of the United

- States Government thereof, and shall not be used for advertising or product endorsement purposes. 